\title{
THE GBC MASS FOR ASYMPTOTICALLY HYPERBOLIC MANIFOLDS
}

\author{
YUXIN GE, GUOFANG WANG, AND JIE WU
}

\begin{abstract}
The paper consists of two parts. In the first part, by using the Gauss-Bonnet curvature, which is a natural generalization of the scalar curvature, we introduce a higher order mass, the Gauss-Bonnet-Chern mass $m_{k}^{\mathbb{H}}$, for asymptotically hyperbolic manifolds and show that it is a geometric invariant. Moreover, we prove a positive mass theorem for this new mass for asymptotically hyperbolic graphs and establish a relationship between the corresponding Penrose type inequality for this mass and weighted Alexandrov-Fenchel inequalities in the hyperbolic space $\mathbb{H}^{n}$. In the second part, we establish these weighted Alexandrov-Fenchel inequalities in $\mathbb{H}^{n}$ for any horospherical convex hypersurface $\Sigma$
\end{abstract}

$$
\int_{\Sigma} V \sigma_{2 k-1} d \mu \geq C_{n-1}^{2 k-1} \omega_{n-1}\left(\left(\frac{|\Sigma|}{\omega_{n-1}}\right)^{\frac{n}{k(n-1)}}+\left(\frac{|\Sigma|}{\omega_{n-1}}\right)^{\frac{n-2 k}{k(n-1)}}\right)^{k}
$$

where $\sigma_{j}$ is the $j$-th mean curvature of $\Sigma \subset \mathbb{H}^{n}, V=\cosh r$ and $|\Sigma|$ is the area of $\Sigma$. As an application, we obtain an optimal Penrose type inequality for the new mass defined in the first part

$$
m_{k}^{\mathbb{H}} \geq \frac{1}{2^{k}}\left(\left(\frac{|\Sigma|}{\omega_{n-1}}\right)^{\frac{n}{k(n-1)}}+\left(\frac{|\Sigma|}{\omega_{n-1}}\right)^{\frac{n-2 k}{k(n-1)}}\right)^{k},
$$

for asymptotically hyperbolic graphs with a horizon type boundary $\Sigma$, provided that a dominant energy condition $\widetilde{L}_{k} \geq 0$ holds. Both inequalities are optimal.

\section{Contents}

\section{Part 1. The GBC mass for asymptotically hyperbolic manifolds}

1. Introduction

2. Preliminaries

2.1. Asymptotically hyperbolic manifolds and the mass for $k=1$

2.2. Gauss-Bonnet curvature and the $k$-th mean curvature

3. A new mass for asymptotically hyperbolic manifolds

4. Positive mass theorem for asymptotically hyperbolic graphs

5. Penrose inequality for graphs over $\mathbb{H}^{n}$ with a horizon type boundary

Appendix A. The anti-de Sitter Schwarzschild type metric

Appendix B. Proof of (3.21)

Part 2. Alexandrov-Fenchel inequalites in $\mathbb{H}^{n}$

6. Introduction

7. Minkowski integral formulas

2000 Mathematics Subject Classification. 53C21, (83C05, 83C30).

Key words and phrases. Gauss-Bonnet-Chern mass, Gauss-Bonnet curvature, asymptotically hyperbolic manifold, positive mass theorem, Penrose inequality, Alexandrov-Fenchel inequality.

This project is partly supported by SFB/TR71 "Geometric partial differential equations" of DFG. 
8. A crucial Minkowski integral formula

9. Weighted Alexandrov-Fenchel inequalities $\quad 36$

References

\section{Part 1. The GBC mass for asymptotically hyperbolic manifolds}

\section{INTRODUCTION}

The study of the scalar curvature plays an important role in differential geometry. There are many beautiful results on manifolds with positive or non-negative scalar curvature. One of them is the Riemannian positive mass theorem (PMT): Any asymptotically flat Riemannian manifold $\mathcal{M}^{n}$ with a suitable decay order and with nonnegative scalar curvature has the nonnegative ADM mass. Moreover, equality holds if and only if the manifold $\mathcal{M}^{n}$ is isometric to the Euclidean space $\mathbb{R}^{n}$ with the standard metric. This theorem was first proved by Schoen and Yau [67] for manifolds of dimension $n \leq 7$ by using a minimal hypersurface argument, and later for spin manifolds by Witten [75. See also [58]. For locally conformally flat manifolds the proof was given in [68] using the developing map. Very recently, for the special case of asymptotically flat Riemannian manifolds $\mathcal{M}^{n}$ which can be represented by graphs over $\mathbb{R}^{n}$, Lam [4] gave a proof by observing that the scalar curvature of these asymptotically flat graphs can be expressed exactly as a divergence term. For general higher dimensional manifolds, the proof of the positive mass theorem was announced by Schoen [66] and Lohkamp [52] by an argument extending the minimal hypersurface argument of Schoen and Yau. There are many generalizations and applications of the positive mass theorem. See [4, 23, 70, 55] for instance.

A refinement of the PMT is the Riemannian Penrose inequality

$$
m_{1}=m_{A D M} \geq \frac{1}{2}\left(\frac{|\Sigma|}{\omega_{n-1}}\right)^{\frac{n-2}{n-1}},
$$

where $m_{A D M}$ is the ADM mass of the asymptotically flat Riemannian manifold with a horizon $\Sigma$ and $|\Sigma|$ denotes the area of $\Sigma$. (1.1) was proved by Huisken-Ilmanen [45] and Bray [8] for $n=3$. Later, Bray and Lee [10] generalized Bray's proof to the case $n \leq 7$. See also the excellent surveys of Bray [9] and Mars [54]. Recently, Lam [47] gave an elegant proof of (1.1) in all dimensions for an asymptotically flat manifold which is a graph. His idea was extended by Huang-Wu in [41] and de Lima-Girão in [24, 25] to submanifolds of $\mathbb{R}^{n+1}$ and of general ambient spaces.

The ADM mass, together with the positive mass theorem, was generalized to asymptotically hyperbolic manifolds in [2, 7, 20, 19, 72, 77]. For this asymptotically hyperbolic mass, the corresponding Penrose conjecture is: For asymptotically hyperbolic manifold $\left(\mathcal{M}^{n}, g\right)$ with an outermost horizon $\Sigma$, its mass satisfies

$$
m_{1}^{\mathbb{H}}=m^{\mathbb{H}} \geq \frac{1}{2}\left\{\left(\frac{|\Sigma|}{\omega_{n-1}}\right)^{\frac{n-2}{n-1}}+\left(\frac{|\Sigma|}{\omega_{n-1}}\right)^{\frac{n}{n-1}}\right\},
$$

provided that the dominant energy condition

$$
R_{g} \geq-n(n-1),
$$


holds. Here $R_{g}$ denotes the scalar curvature of $g$. Neves [57] showed that the powerful inverse mean curvature flow of Huisken-Ilmanen [45] alone could not be used to prove (1.2). (However, very recently Lee and Neves have a new development in this direction [49.) Recently, motivated by the work of Lam [47, Dahl-Gicquaud-Sakovich [22] and de Lima and Girão [26] proved the Penrose inequality (1.2) for asymptotically hyperbolic graphs over $\mathbb{H}^{n}$. More precisely, DahlGicquaud-Sakovich [22] proved for asymptotically hyperbolic graphs generated by a function $f: \mathbb{H}^{n} \backslash \Omega \rightarrow \mathbb{R}$, the hyperbolic mass satisfies a crucial estimate in terms of a weighted mean curvature integral

$$
m_{1}^{\mathbb{H}} \geq \frac{1}{2(n-1) \omega_{n-1}} \int_{\Sigma} V H d \mu
$$

where $\Sigma=\partial \Omega, H$ is the mean curvature of $\Sigma$ induced by the hyperbolic metric $b, \omega_{n-1}$ is the area of the unit sphere, $d \mu$ is the volume element induced by $b, V=\cosh r$ and $r$ is the hyperbolic distance from an arbitrary fixed point on $\mathbb{H}^{n}$. They also gave an almost sharp estimate for $\int_{\Sigma} V H d \mu$, and hence an almost sharp estimate for the hyperbolic mass. The sharp estimate for $\int_{\Sigma} V H d \mu$ is a weighted hyperbolic Minkowski inequality, or a weighted hyperbolic Alexandrov-Fenchel inequality

$$
\int_{\Sigma} V H d \mu \geq(n-1) \omega_{n-1}\left\{\left(\frac{|\Sigma|}{\omega_{n-1}}\right)^{\frac{n-2}{n-1}}+\left(\frac{|\Sigma|}{\omega_{n-1}}\right)^{\frac{n}{n-1}}\right\},
$$

if $\Sigma$ is star-shaped and mean-convex (i.e. $H>0$ ). This result was proved by de Lima and Girão [26]. A closely related Minkowski type inequality was proved by Brendle-Hung-Wang [12, which is not only true for the hyperbolic space, but also for anti-de Sitter Schwarzschild manifolds. For the proof of (1.5), a Heintze-Karcher type inequality proved by Brendle in [11] and an inverse curvature flow studied by Gerhardt [34] play an important role.

Recently motivated by the Gauss-Bonnet gravity [27, 28], we have introduced the GaussBonnet-Chern mass $m_{G B C}$ for asymptotically flat manifolds by using the following Gauss-Bonnet curvature

$$
L_{k}:=\frac{1}{2^{k}} \delta_{j_{1} j_{2} \cdots j_{2 k-1} j_{2 k}}^{i_{1} i_{2} \cdots i_{2 k-1} i_{2 k}} R_{i_{1} i_{2}}^{{ }^{j} j_{2}} \cdots R_{i_{2 k-1} i_{2 k}}^{j_{2 k-1} j_{2 k}} .
$$

Here $\delta_{j_{1} j_{2} \cdots j_{2 k-1} j_{2 k}}^{i_{1} i_{2} \cdots i_{2 k-1} i_{2 k}}$ is the generalized delta defined by (2.9) below and $R_{i j}{ }^{s l}$ is the Riemannian curvature tensor. One can check that $L_{1}$ is just the scalar curvature $R$. When $k=2$, it is the (second) Gauss-Bonnet curvature

$$
L_{2}=\|R m\|^{2}-4\|R i c\|^{2}+R^{2},
$$

which appeared at the first time in the paper of Lanczos [48] in 1938. For general $k$, it is just the Euler integrand in the Gauss-Bonnet-Chern theorem [17, 18, if $n=2 k$ and is therefore called the dimensional continued Euler density in physics if $k<n / 2$. A systematic study of $L_{k}$ was first given by Lovelock [53. See also [59, [74] and [46]. The Gauss-Bonnet-Chern mass $m_{G B C}$ for the asymptotically flat manifolds is defined in [30] by

$$
m_{k}=m_{G B C}=c(n, k) \lim _{r \rightarrow \infty} \int_{S_{r}} P_{(k)}^{i j l s} \partial_{s} g_{j l} \nu_{i} d \mu,
$$

with

$$
c(n, k)=\frac{(n-2 k) !}{2^{k-1}(n-1) ! \omega_{n-1}},
$$


where $\omega_{n-1}$ is the volume of $(n-1)$-dimensional standard unit sphere and $S_{r}$ is the Euclidean coordinate sphere, $d \mu$ is the volume element on $S_{r}$ induced by the Euclidean metric and $\nu$ is the outward unit normal to $S_{r}$ in $\mathbb{R}^{n}$. Here the $(0,4)$-tensor $P_{(k)}$ is defined in (2.11) below. This $(0,4)$-tensor has a crucial property that it is divergence-free. See Section 2 below. For a similar definition see also [50]. In [30] and [31, we prove a positive mass theorem in the case that $\mathcal{M}$ is an asymptotically flat graph over $\mathbb{R}^{n}$ or $\mathcal{M}$ is conformal to $\mathbb{R}^{n}$ respectively. For our mass $m_{G B C}$, a corresponding Penrose conjecture was proposed in [30]

$$
m_{k}=m_{G B C} \geq \frac{1}{2^{k}}\left(\frac{|\Sigma|}{\omega_{n-1}}\right)^{\frac{n-2 k}{n-1}} .
$$

Moreover we proved in [30] that this conjecture is true for asymptotically flat graphs over $\mathbb{R}^{n} \backslash \Omega$ by using classical Alexandrov-Fenchel inequalities, if $\Sigma=\partial \Omega$ is convex. For the classical Alexandrov-Fenchel inequalities see excellent books [13, 63, 65]. The Alexandrov-Fenchel inequalities also hold for certain classes of non-convex hypersurfaces. See for example [15, 36, 44].

In this paper, motivated by our previous work, by using the Gauss-Bonnet curvature we introduce a higher order mass for asymptotically hyperbolic manifolds, which is a generalization of the mass introduced by Wang [72] and Cruściel-Herzlich [19]. See also [20, 40, 56, 77]. However, if we use directly the Gauss-Bonnet curvature $L_{k}$, we can only obtain a mass proportional to the the usual hyperbolic mass, rather than a new one. This phenomenon is different from the Euclidean case. In order to define a higher order mass for asymptotically hyperbolic manifolds, the crucial observation is a slight modification of the Gauss-Bonnet curvature. More precisely, on a Riemannian manifold $\left(\mathcal{M}^{n}, g\right)$, we consider a modified Riemann curvature tensor

$$
\widetilde{\operatorname{Riem}}_{i j l s}(g)=\widetilde{R}_{i j l s}(g):=R_{i j l s}(g)+g_{i l} g_{j s}-g_{i s} g_{j l} .
$$

Clearly, the modified Riemann curvature tensor $\widetilde{\text { Riem }}$ has the same symmetry as the ordinary Riemann curvature tensor Riem and also satisfies the first and second Bianchi identities. We define a new Gauss-Bonnet curvature with respect to this tensor $\overparen{\text { Riem }}$

$$
\widetilde{L}_{k}:=\frac{1}{2^{k}} \delta_{j_{1} j_{2} \cdots j_{2 k-1} j_{2 k}}^{i_{1} i_{2} \cdots i_{2 k-1} i_{2 k}} \widetilde{R}_{i_{1} i_{2}}^{j_{1} j_{2}} \cdots \widetilde{R}_{i_{2 k-1} i_{2 k}}^{j_{2 k-1} j_{2 k}}=\widetilde{R}_{i j l s} \widetilde{P}_{(k)}^{i j l s},
$$

where

$$
\widetilde{P}_{(k)}^{i j l s}:=\frac{1}{2^{k}} \delta_{j_{1} j_{2} \cdots j_{2 k-3} j_{2 k-2} j_{2 k-1} j_{2 k}}^{i_{1} i_{2} \cdots i_{2 k-3} i_{2 k-2} i j} \widetilde{R}_{i_{1} i_{2}}^{j_{1} j_{2}} \cdots \widetilde{R}_{i_{2 k-3} i_{2 k-2}}^{j_{2 k-3} j_{2 k-2}} g^{j_{2 k-1} l} g^{j_{2 k} s} .
$$

The tensor $\widetilde{P}_{(k)}$ has also the crucial property of divergence-free which enables us to define a new mass.

Let us assume now that $2 \leq k<\frac{n}{2}$. Remark that the case $k=1$ was studied in [72, 19, 77, 2 , 20, 22, 24, 26, 42, 12, 51 mentioned above and we will not repeat this remark again later.

Let us first give the definition of asymptotically hyperbolic manifolds. Throughout this paper, we denote the hyperbolic metric by $\left(\mathbb{H}^{n}, b=d r^{2}+\sinh ^{2} r d \Theta^{2}\right)$, where $d \Theta^{2}$ is the standard round metric on $\mathbb{S}^{n-1}$.

A Riemannian manifold $\left(\mathcal{M}^{n}, g\right)$ is called asymptotically hyperbolic of decay order $\tau$ if there exists a compact subset $K$ and a diffeomorphism at infinity $\Phi: \mathcal{M} \backslash K \rightarrow \mathbb{H}^{n} \backslash B$, where $B$ is a closed ball in $\mathbb{H}^{n}$, such that $\left(\Phi^{-1}\right)^{*} g$ and $b$ are uniformally equivalent on $\mathbb{H}^{n} \backslash B$ and

$$
\left\|\left(\Phi^{-1}\right)^{*} g-b\right\|_{b}+\left\|\bar{\nabla}\left(\left(\Phi^{-1}\right)^{*} g\right)\right\|_{b}+\left\|\bar{\nabla}^{2}\left(\left(\Phi^{-1}\right)^{*} g\right)\right\|_{b}=O\left(e^{-\tau r}\right),
$$

where $\bar{\nabla}$ denotes the covariant derivative with respect to the hyperbolic metric $b$. 
In the first part of this paper we first introduce a "higher order" mass for asymptotically hyperbolic manifolds with slower decay.

Definition 1.1. Assume that $\left(\mathcal{M}^{n}, g\right)$ is an asymptotically hyperbolic manifold of decay order

$$
\tau>\frac{n}{k+1},
$$

and $V \widetilde{L}_{k}$ is integrable on $\left(\mathcal{M}^{n}, g\right)$ for $V \in \mathbb{N}_{b}:=\left\{V \in C^{\infty}\left(\mathbb{H}^{n}\right) \mid \operatorname{Hess}^{b} V=V b\right\}$. We define the Gauss-Bonnet-Chern mass integral with respect to the diffeomorphism $\Phi$ by

$$
H_{k}^{\Phi}(V)=\lim _{r \rightarrow \infty} \int_{S_{r}}\left(\left(V \bar{\nabla}_{s} e_{j l}-e_{j l} \bar{\nabla}_{s} V\right) \widetilde{P}_{(k)}^{i j l s}\right) \nu_{i} d \mu,
$$

where $e_{i j}:=\left(\left(\Phi^{-1}\right)^{*} g\right)_{i j}-b_{i j}$.

Remark that the hyperbolic mass $m_{1}^{\mathbb{H}}$ defined by Wang [72] and Cruściel-Herzlich [19] can in principle be defined for asymptotically hyperbolic manifolds of decay order

$$
\tau>\frac{n}{2}
$$

For a discussion about the range of $\tau$, see Remark 3.4 below.

The above definition of the asymptotically hyperbolic mass involves the choice of coordinates at infinity. Hence one needs to ask if it is a geometric invariant, namely if it does not depend on the choice of coordinates at infinity. This is true.

Theorem 1.2. Suppose that $\left(\mathcal{M}^{n}, g\right)$ is an asymptotically hyperbolic manifold of decay order $\tau>\frac{n}{k+1}$ and for $V \in \mathbb{N}_{b}, V \widetilde{L}_{k}$ is integrable on $\left(\mathcal{M}^{n}, g\right)$, then the mass functional $H_{k}^{\Phi}(V)$ is welldefined and does not depend on the choice of the coordinates at infinity used in the definition.

The proof is motivated by [19] and [56] and certainly also by [4]. From the mass functional $H_{k}^{\Phi}$ on $\mathbb{N}_{b}$ we define a higher order mass, the Gauss-Bonnet-Chern mass for asymptotically hyperbolic manifolds as follows

$$
m_{k}^{\mathbb{H}}:=c(n, k) \inf _{\mathbb{N}_{b} \cap\{V>0, \eta(V, V)=1\}} H_{k}^{\Phi}(V),
$$

where $c(n, k)$ is the normalization constant given in (1.8) and $\eta$ is a Lorentz inner product defined in (2.3) below. A more precise definition will be given in Definition 3.3. As discussed in (3.25) below, one may assume that the infimum in (1.15) is achieved by

$$
V=V_{(0)}=\cosh r
$$

where $r$ is the hyperbolic distance to a fixed point $x_{0} \in \mathbb{H}^{n}$. Therefore, in the rest of the introduction, we fix $V=V_{(0)}=\cosh r$.

Theorem 1.3 (Positive Mass Theorem). Let $\left(\mathcal{M}^{n}, g\right)=\left(\mathbb{H}^{n}, b+V^{2} d f \otimes d f\right)$ be the graph of a smooth function $f: \mathbb{H}^{n} \rightarrow \mathbb{R}$ which satisfies $V \widetilde{L}_{k}$ is integrable and $\left(\mathcal{M}^{n}, g\right)$ is asymptotically hyperbolic of decay order $\tau>\frac{n}{k+1}$. Then we have

$$
m_{k}^{\mathbb{H}}=c(n, k) \int_{\mathcal{M}^{n}} \frac{1}{2} \frac{V \widetilde{L}_{k}}{\sqrt{1+V^{2}|\bar{\nabla} f|^{2}}} d V_{g} .
$$

In particular, $\widetilde{L}_{k} \geq 0$ implies $m_{k}^{\mathbb{H}} \geq 0$. 
The condition

$$
\widetilde{L}_{k} \geq 0
$$

is a dominant energy condition like (1.3). In fact, one can check when $k=1, \widetilde{L}_{1}=R+n(n-1)$. When $k=2, \widetilde{L}_{2}=L_{2}+2(n-2)(n-3) R+n(n-1)(n-2)(n-3)$. By the definition of $\widetilde{L}_{k}$ (see (1.11) and (1.10) $)$ it is trivial to see that $\widetilde{L}_{k}(b)$ of the standard hyperbolic metric $b$ vanishes for all $k$. Hence (1.17) is the same as $\widetilde{L}_{k} \geq \widetilde{L}_{k}(b)$.

Such a beautiful expression (1.16) was found first by Lam for the ADM mass for asymptotically flat graphs over $\mathbb{R}^{n}$, and was generalized for the Gauss-Bonnet-Chern mass in [30]. DahlGicquaud-Sakovich [22] obtained this formula for $m_{1}^{\mathbb{H}}$ for asymptotically hyperbolic graphs in $\mathbb{H}^{n}$. See also the work of de Lima-Girão [26] and Huang-Wu [41].

Furthermore, if the manifold is an asymptotically hyperbolic graph with a horizon boundary, we establish a relationship between our new mass and a weighted higher order mean curvature in the following.

Theorem 1.4. Let $\Omega$ be a bounded open set in $\mathbb{H}^{n}$ with boundary $\Sigma=\partial \Omega$, and let $\left(\mathcal{M}^{n}, g\right)=$ $\left(\mathbb{H}^{n} \backslash \Omega, b+V^{2} d f \otimes d f\right)$ an asymptotically hyperbolic graph of decay order $\tau>\frac{n}{k+1}$ with a horizon boundary $\Sigma$ satisfying that $V \widetilde{L}_{k}$ is integrable over $\mathcal{M}^{n}$. Assume that each connected component of $\Sigma$ is in a level set of $f$ and $|\bar{\nabla} f(x)| \rightarrow \infty$ as $x \rightarrow \Sigma$. Then

$$
m_{k}^{\mathbb{H}}=c(n, k)\left(\frac{1}{2} \int_{\mathcal{M}^{n}} \frac{V \widetilde{L}_{k}}{\sqrt{1+V^{2}|\nabla f|^{2}}} d V_{g}+\frac{(2 k-1) !}{2} \int_{\Sigma} V \sigma_{2 k-1} d \mu\right),
$$

where $\sigma_{k}$ denotes $k$-th mean curvature of $\Sigma$ induced by the hyperbolic metric $b$.

For the precise definition of asymptotically hyperbolic graphs with a horizon boundary, see Section 5 below.

In order to obtain a Penrose type inequality for the hyperbolic mass $m_{k}^{\mathbb{H H}}$ for asymptotically hyperbolic graphs with a horizon, we need to establish a "weighted" hyperbolic AlexandrovFenchel inequality. This is the objective of the second part of this paper. To state this inequality, let us first introduce a definition. A hypersurface in $\mathbb{H}^{n}$ is horospherical convex if all principal curvatures are larger than or equal to 1 . The horospherical convexity is a natural geometric concept, which is equivalent to the geometric convexity in the hyperbolic space.

Theorem 1.5. Let $\Sigma$ be a horospherical convex hypersurface in the hyperbolic space $\mathbb{H}^{n}$. We have

$$
\int_{\Sigma} V \sigma_{2 k-1} d \mu \geq C_{n-1}^{2 k-1} \omega_{n-1}\left(\left(\frac{|\Sigma|}{\omega_{n-1}}\right)^{\frac{n}{k(n-1)}}+\left(\frac{|\Sigma|}{\omega_{n-1}}\right)^{\frac{n-2 k}{k(n-1)}}\right)^{k} .
$$

Equality holds if and only if $\Sigma$ is a geodesic sphere centered at $x_{0}$ in $\mathbb{H}^{n}$.

When $k=1$, inequality (1.18) is just (1.5), which was proved by de Lima and Girão in [26]. These inequalities have their own interest in integral geometry as well as in differential geometry. Recently, another type of Alexandrov-Fenchel inequalities in $\mathbb{H}^{n}$ without the weighted $V$ has been established in [51, 32, 33, 73]. We will give a short introduction about Alexandrov-Fenchel inequalities in Section 6 below.

As a consequence of Theorem 1.4 and Theorem [1.5, the Penrose inequality for the GaussBonnet-Chern mass $m_{k}^{\mathbb{H}}$ for asymptotically hyperbolic graphs with horizon boundaries follows. 
Theorem 1.6 (Penrose Inequality). Assume that all conditions given in Theorem 1.4 hold. If each connected component of $\Sigma$ is horospherical convex, then

$$
m_{k}^{\mathbb{H}} \geq \frac{1}{2^{k}}\left(\left(\frac{|\Sigma|}{\omega_{n-1}}\right)^{\frac{n}{k(n-1)}}+\left(\frac{|\Sigma|}{\omega_{n-1}}\right)^{\frac{n-2 k}{k(n-1)}}\right)^{k}
$$

provided that

$$
\widetilde{L}_{k} \geq 0
$$

Moreover, equality is achieved by the anti-de Sitter Schwarzschild type metric

$$
g_{\text {adS-Sch }}=\left(1+\rho^{2}-\frac{2 m}{\rho^{\frac{n}{k}-2}}\right)^{-1} d \rho^{2}+\rho^{2} d \Theta^{2}, \quad \rho=\sinh r .
$$

Remark that metric (1.20) can be represented as a graph in $\mathbb{H}^{n+1}$. See Section 5 below. Motivated by the previous results, it is natural to propose the following conjecture.

Conjecture 1.7. Let $k<\frac{n}{2}$ and let $\mathcal{M}^{n}$ be a complete asymptotically hyperbolic manifold of decay order $\tau>\frac{n}{k+1}$ and with finite integral $\int_{\mathcal{M}} V\left|\widetilde{L}_{k}\right| d \mu$. Assume that the dominant energy condition

$$
\widetilde{L}_{k} \geq 0
$$

holds, then the new mass for asymptotically hyperbolic manifolds

$$
m_{k}^{\mathbb{H}} \geq 0 \text {. }
$$

Moreover, if $\mathcal{M}$ has a horizon type boundary $\Sigma$, then the Penrose inequality

$$
m_{k}^{\mathbb{H}} \geq \frac{1}{2^{k}}\left(\left(\frac{|\Sigma|}{\omega_{n-1}}\right)^{\frac{n}{k(n-1)}}+\left(\frac{|\Sigma|}{\omega_{n-1}}\right)^{\frac{n-2 k}{k(n-1)}}\right)^{k},
$$

holds. Furthermore, the rigidity theorem holds. More precisely, equality in (1.21) implies that $\mathcal{M}$ is isometric to the standard hyperbolic space $\mathbb{H}^{n}$ and equality in (1.22) implies that $\mathcal{M}$ is isometric to the anti de-Sitter Schwarzschild type metric (1.20) outside its corresponding horizon.

The rest of this paper is organized as follows. In Section 2, some preliminaries are reviewed. This section is divided into two subsections. In Subsection 2.1, we recall the mass of asymptotically hyperbolic manifolds defined in [19, 20. In Subsection 2.2, some basic facts of the Gauss-Bonnet curvature as well as some basic properties of the $k$-th mean curvature are outlined. In Section 3, we introduce the higher order mass for asymptotically hyperbolic manifolds with slower decay by using the Gauss-Bonnet curvature. Its geometric invariance is also proved in Section 3. For this new defined mass, we show a positive mass theorem for asymptotically hyperbolic graphs in Section 4 and establish a relationship between the corresponding Penrose type inequality and "weighted" Alexandrov-Fenchel inequalities in the hyperbolic space in Section 5. Moreover, an interesting example, a Schwarzschild type metric, is also given in this section. In Section 6, we give a short introduction for the Alexandrov-Fenchel inequalities with or without weight in the hyperbolic space. As a preparation to establish these Alexandrov-Fenchel inequalities, several important Minkowski integral formulas between integrals involving $\sigma_{k}$ are proved in Section 7. Section 8 is devoted to discuss our crucial Minkowski integral formulas between integrals involving $\sigma_{k}$ with the weight (see (8.5) below) by using a "conformal" flow. We establish "weighted" Alexandrov-Fenchel inequalities in Section 9, which implies optimal Penrose 
type inequalities for the new mass for asymptotically hyperbolic graphs with a horizon type boundary. We include calculations of the modified Gauss-Bonnet curvature $\widetilde{L}_{k}$ for the anti-de Sitter-Schwarzschild type metric (1.20) as well as its Gauss-Bonnet-Chern mass $m_{k}^{\mathbb{H I}}$ in Appendix A. We show formula (3.21) in Appendix B.

\section{Preliminaries}

2.1. Asymptotically hyperbolic manifolds and the mass for $k=1$. In this subsection, we recall the mass of asymptotically hyperbolic manifolds from Chruściel and Herzlich [19] which coincides with the one defined by Wang [72] for the special case of conformally compact manifolds. Throughout this paper, we denote the hyperbolic metric by $\left(\mathbb{H}^{n}, b=d r^{2}+\sinh ^{2} r d \Theta^{2}\right)$, where $d \Theta^{2}$ is the standard round metric on $\mathbb{S}^{n-1}$. We recall the definition of asymptotically hyperbolic manifolds.

Definition 2.1. A Riemannian manifold $\left(\mathcal{M}^{n}, g\right)$ is called asymptotically hyperbolic of decay order $\tau$ if there exists a compact subset $K$ and a diffeomorphism at infinity $\Phi: \mathcal{M} \backslash K \rightarrow \mathbb{H}^{n} \backslash B$, where $B$ is a closed ball in $\mathbb{H}^{n}$, such that $\left(\Phi^{-1}\right)^{*} g$ and $b$ are uniformly equivalent on $\mathbb{H}^{n} \backslash B$ and

$$
\left\|\left(\Phi^{-1}\right)^{*} g-b\right\|_{b}+\left\|\bar{\nabla}\left(\left(\Phi^{-1}\right)^{*} g\right)\right\|_{b}+\left\|\bar{\nabla}^{2}\left(\left(\Phi^{-1}\right)^{*} g\right)\right\|_{b}=O\left(e^{-\tau r}\right) .
$$

Note that when $k=1$, one needs no decay condition on the second derivatives of $\left(\Phi^{-1}\right)^{*} g$, see [19]. Set

$$
\mathbb{N}_{b}:=\left\{V \in C^{\infty}\left(\mathbb{H}^{n}\right) \mid \operatorname{Hess}^{b} V=V b\right\} .
$$

Any element $V$ in $\mathbb{N}_{b}$ has a nice property that the Lorentzian metric $\gamma=-V^{2} d t^{2}+b$ is a static solution of the Einstein equation $\operatorname{Ric}(\gamma)+n \gamma=0 . \mathbb{N}_{b}$ is an $(n+1)$-dimensional vector space spanned by an orthonormal basis of the following functions

$$
V_{(0)}=\cosh r, V_{(1)}=x^{1} \sinh r, \cdots, V_{(n)}=x^{n} \sinh r,
$$

where $r$ is the hyperbolic distance from an arbitrary fixed point on $\mathbb{H}^{n}$ and $x^{1}, x^{2}, \cdots, x^{n}$ are the coordinate functions restricted to $\mathbb{S}^{n-1} \subset \mathbb{R}^{n}$. We equip the vector space $\mathbb{N}_{b}$ with a Lorentz inner product $\eta$ with signature $(+,-, \cdots,-)$ such that

$$
\eta\left(V_{(0)}, V_{(0)}\right)=1, \quad \text { and } \quad \eta\left(V_{(i)}, V_{(i)}\right)=-1 \quad \text { for } \quad i=1, \cdots, n .
$$

It is clear that the subset $\mathbb{N}_{b}^{+}$of positive functions is just the interior of the future lightcone. Let $\mathbb{N}_{b}^{1}$ be the subset of $\mathbb{N}_{b}^{+}$of functions $V$ with $\eta(V, V)=1$. One can check easily that every function $V$ in $\mathbb{N}_{b}^{1}$ has the following form

$$
V=\cosh _{\operatorname{dist}}\left(x_{0}, \cdot\right),
$$

for some $x_{0} \in \mathbb{H}^{n}$, where dist $_{b}$ is the distance function with respect to the metric $b$.

Let $\left(\mathcal{M}^{n}, g\right)$ be an asymptotically hyperbolic manifold of decay order $\tau>\frac{n}{2}$ and

$$
\int_{\mathcal{M}} \cosh r|R+n(n-1)| d \mu<\infty
$$

where $R$ is the scalar curvature with respect to the metric $g$. Then the mass functional of $\left(\mathcal{M}^{n}, g\right)$ with respect to $\Phi$ on $\mathbb{N}_{b}$ is defined by

$$
H_{\Phi}(V)=\lim _{r \rightarrow \infty} \int_{S_{r}}\left(V\left(\operatorname{div}^{b} e-d \operatorname{tr}^{b} e\right)+\left(\operatorname{tr}^{b} e\right) d V-e\left(\nabla^{b} V, \cdot\right)\right) \nu d \mu
$$


where $e:=\Phi_{*} g-b, S_{r}$ is a geodesic sphere with radius $r, \nu$ is the outer normal of $S_{r}$ and $d \mu$ is the area element with respect to the induced metric on $S_{r}$. From[19], the limit in (2.5) exists and is finite, provided that the decay condition $\tau>\frac{n}{2}$ and the integrable condition (2.4) holds. If $A$ is an isometry of the hyperbolic metric $b$ and $\Phi$ a chart as defined in Definition 2.1, then one can easily check that $A \circ \Phi$ is also such a chart and

$$
H_{A \circ \Phi}(V)=H_{\Phi}\left(V \circ A^{-1}\right) \text {, }
$$

holds. Moreover, if $\Psi$ is another chart satisfying conditions in Definition 2.1, there is an isometry $A$ of $b$ such that $\Psi=A \circ \Phi$ modulo lower order terms [19, 40] such that the mass functional is not changed. It follows that the defined limit (2.5) is a geometric invariant independent of the choice of coordinates at infinity. See also Theorem 3.3 in [56]. If the mass functional $H_{\Phi}: \mathbb{N}_{b} \rightarrow \mathbb{R}$ is timelike future directed, i.e., $H_{\Phi}(V)>0$ for all $V \in \mathbb{N}^{+}$, then we can define the asymptotically hyperbolic mass $m^{\mathbb{H}}$ for asymptotically hyperbolic manifold of decay order $\tau>\frac{n}{2}$ as follows:

$$
m_{1}^{\mathbb{H}}:=\frac{1}{2(n-1) \omega_{n-1}} \inf _{\mathbb{N}_{b}^{1}} H_{\Phi}(V) .
$$

From above discussion we know that

$$
m_{1}^{\mathbb{H}}=\frac{1}{2(n-1) \omega_{n-1}} \inf _{\Phi} H_{\Phi}\left(V_{(0)}\right)
$$

where the infimum takes over of asymptotically hyperbolic charts $\Phi$ as above. Hence to estimate $m^{\mathbb{H I}}$ we only need to estimate $H_{\Phi}\left(V_{(0)}\right)$ for a fixed $V_{(0)}$ and for all $\Phi$ and $V_{(0)}$ is a fixed function in $\mathbb{N}_{b}^{1}$. This strategy will also be used to estimate our new mass defined in Section 3 below.

2.2. Gauss-Bonnet curvature and the $k$-th mean curvature. Recall that

$$
L_{k}:=\frac{1}{2^{k}} \delta_{j_{1} j_{2} \cdots j_{2 k-1} j_{2 k}}^{i_{1} i_{2} \cdots i_{2 k-1} i_{2 k}} R_{i_{1} i_{2}}{ }^{j_{1} j_{2}} \cdots R_{i_{2 k-1} i_{2 k}}^{j_{2 k-1} j_{2 k}}
$$

is the $k$-th Gauss-Bonnet curvature, or simply the Gauss-Bonnet curvature. Here the generalized Kronecker delta is defined by

$$
\delta_{i_{1} i_{2} \cdots i_{r}}^{j_{1} j_{2} \cdots j_{r}}=\operatorname{det}\left(\begin{array}{cccc}
\delta_{i_{1}}^{j_{1}} & \delta_{i_{1}}^{j_{2}} & \cdots & \delta_{i_{1}}^{j_{r}} \\
\delta_{i_{2}}^{j_{1}} & \delta_{i_{2}}^{j_{2}} & \cdots & \delta_{i_{2}}^{j_{r}} \\
\vdots & \vdots & \vdots & \vdots \\
\delta_{i_{r}}^{j_{1}} & \delta_{i_{r}}^{j_{2}} & \cdots & \delta_{i_{r}}^{j_{r}}
\end{array}\right) .
$$

This curvature was first appeared in the paper of Lanczos [48] in 1938 for $n=4$ and $k=2$ and has been intensively studied in the Gauss-Bonnet gravity, which is a generalization of the Einstein gravity. $L_{1}$ is just the scalar curvature. One could check that $L_{k}=0$ if $2 k>n$. When $2 k=n, L_{k}$ is in fact the Euler density, which was studied by Chern [17, 18] in his proof of the Gauss-Bonnet-Chern theorem. See also a nice survey [76] on the proof of the Gauss-BonnetChern theorem. For $k<n / 2, L_{k}$ is therefore called the dimensional continued Euler density in physics. One can decompose the Gauss-Bonnet curvature in the following way

$$
L_{k}=P_{(k)}^{s t j l} R_{s t j l}
$$

where

$$
P_{(k)}^{s t l j}:=\frac{1}{2^{k}} \delta_{j_{1} j_{2} \cdots j_{2 k-3} j_{2 k-2} j_{2 k-1} j_{2 k}}^{i_{1} i_{2} \cdots i_{2 k-3} i_{2 k-2} s t} R_{i_{1} i_{2}}^{j_{1} j_{2}} \cdots R_{i_{2 k-3} i_{2 k-2}}^{j_{2 k-3} j_{2 k-2}} g^{j_{2 k-1} l} g^{j_{2 k} j}
$$


(Throughout this paper, we adopt the Einstein summation convention.) For example, for the case $k=1$,

$$
L_{1}=R=R_{s t j l} P_{(1)}^{s t j l}
$$

where

$$
P_{(1)}^{s t j l}=\frac{1}{2}\left(g^{s j} g^{t l}-g^{s l} g^{t j}\right)
$$

For the case $k=2$, we have

$$
L_{2}=\frac{1}{4} \delta_{j_{1} j_{2} j_{3} j_{4}}^{i_{1} i_{2} i_{3} i_{4}} R_{{ }_{1} i_{2}}^{j_{1} j_{2}} R_{i_{3} i_{4}}^{j_{3} j_{4}}=\|R m\|^{2}-4\|R i c\|^{2}+R^{2}
$$

and

$$
P_{(2)}^{s t j l}=R^{s t j l}+R^{t j} g^{s l}-R^{t l} g^{s j}-R^{s j} g^{t l}+R^{s l} g^{t j}+\frac{1}{2} R\left(g^{s j} g^{t l}-g^{s l} g^{t j}\right) .
$$

We collect important properties of the four-tensor $P_{(k)}$ in the following lemma.

Lemma 2.2. (1) $P_{(k)}$ shares the same symmetry and antisymmetry with the Riemann curvature tensor that

$$
P_{(k)}^{s t j l}=-P_{(k)}^{t s j l}=-P_{(k)}^{s t l j}=P_{(k)}^{j l s t} .
$$

(2) $P_{(k)}$ satisfies the first Bianchi identity, i.e., $P^{s t j l}+P^{t j s l}+P^{j s t l}=0$.

(3) $P_{(k)}$ is divergence-free,

$$
\nabla_{s} P_{(k)}^{s t j l}=0
$$

Proof. (1) is easy to check. For (2), one can also calculate it directly, or see page 26 in [74]. Here we provide a proof for (3), since this property is crucial. To show (2.17), we use the differential Bianchi identity

$$
\nabla_{s} R_{i_{1} i_{2}}{ }^{j_{1} j_{2}}=-\nabla_{i_{1}} R_{i_{2} s}{ }^{{ }_{1} j_{2}}-\nabla_{i_{2}} R_{s i_{1}}{ }^{j_{1} j_{2}},
$$

and the fact of the symmetry property of the generalized Kronecker delta and the curvature tensor. From (2.11), we compute

$$
\begin{aligned}
& \nabla_{s} P_{(k)}^{s t j l} \\
= & \frac{k-1}{2^{k}} \delta_{j_{1} j_{2} \cdots j_{2 k-3} j_{2 k-2} j_{2 k-1} j_{2 k}}^{i_{1} i_{2} \cdots i_{2 k-3} i_{2 k-2} s t}\left(\nabla_{s} R_{i_{1} i_{2}}{ }^{j_{1} j_{2}}\right) \cdots R_{i_{2 k-3} i_{2 k-2}}{ }^{j_{2 k-3} j_{2 k-2}} g^{j_{2 k-1} j} g^{j_{2 k} l} \\
= & \frac{k-1}{2^{k}} \delta_{j_{1} j_{2} \cdots j_{2 k-3} j_{2 k-2} j_{2 k-1} j_{2 k}}^{i_{1} i_{2} \cdots i_{2 k-3} i_{2 k-2} s t}\left(-\nabla_{i_{1}} R_{i_{2} s}{ }^{j_{1} j_{2}}-\nabla_{i_{2}} R_{s i_{1}}{ }^{j_{1} j_{2}}\right) \cdots R_{i_{2 k-3} i_{2 k-2}}{ }^{j_{2 k-3} j_{2 k-2} g^{j_{2 k-1} j} g^{j_{2 k} l}} \\
= & \frac{k-1}{2^{k}} \delta_{j_{1} j_{2} \cdots j_{2 k-3} j_{2 k-2} j_{2 k-1} j_{2 k}}^{i_{1} i_{2} \cdots i_{2 k-3} i_{2 k-2} s t}\left(-2 \nabla_{i_{1}} R_{i_{2} s}{ }^{j_{1} j_{2}}\right) \cdots R_{i_{2 k-3} i_{2 k-2}}{ }^{j_{2 k-3} j_{2 k-2}} g^{j_{2 k-1} j} g^{j_{2 k} l} \\
= & \frac{k-1}{2^{k}} \delta_{j_{1} j_{2} \cdots j_{2 k-3} j_{2 k-2} j_{2 k-1} j_{2 k}}^{i_{1} i_{2} \cdots i_{2 k-3} i_{2 k-2} s t}\left(-2 \nabla_{s} R_{i_{1} i_{2}}{ }^{j_{1} j_{2}}\right) \cdots R_{i_{2 k-3} i_{2 k-2}}^{j_{2 k-3} j_{2 k-2}} g^{j_{2 k-1} j} g^{j_{2 k} l} \\
= & -2 \nabla_{s} P_{(k)}^{s t j l},
\end{aligned}
$$

which implies

$$
\nabla_{s} P_{(k)}^{s t j l}=0
$$

Thus we complete the proof. 
This Lemma, in particular, the divergence-free property (2.17), plays a crucial role in this paper.

In the following, we recall several basic properties of the $k$-th mean curvature. Let $\sigma_{k}$ be the $k$-th elementary symmetry function $\sigma_{k}: \mathbb{R}^{n-1} \rightarrow \mathbb{R}$ defined by

$$
\sigma_{k}(\Lambda)=\sum_{i_{1}<\cdots<i_{k}} \lambda_{i_{1}} \cdots \lambda_{i_{k}} \quad \text { for } \Lambda=\left(\lambda_{1}, \cdots, \lambda_{n-1}\right) \in \mathbb{R}^{n-1} .
$$

This definition can be easily extended to symmetric matrices. For a symmetric matrix $B$, denote $\lambda(B)=\left(\lambda_{1}(B), \cdots, \lambda_{n-1}(B)\right)$ be the eigenvalues of $B$. We set

$$
\sigma_{k}(B):=\sigma_{k}(\lambda(B)) .
$$

Here we make a convention that $\sigma_{0}(B)=1$. Let $I$ be the identity matrix. Then we have for any $t \in \mathbb{R}$

$$
\sigma_{n-1}(I+t B)=\operatorname{det}(I+t B)=\sum_{i=0}^{n-1} \sigma_{i}(B) t^{i} .
$$

The Garding cone $\Gamma_{k}^{+}$is defined as

$$
\Gamma_{k}^{+}=\left\{\Lambda \in \mathbb{R}^{n-1} \mid \sigma_{j}(\Lambda)>0, \quad \forall j \leq k\right\} .
$$

A symmetric matrix $B$ is called belong to $\Gamma_{k}^{+}$if $\lambda(B) \in \Gamma_{k}^{+}$. We collect the basic facts about $\sigma_{k}$, which will be directly used in this paper. For further details, we refer to a survey of Guan [35].

The $k$-th Newton transformation is defined as follows

$$
\left(T_{k}\right)_{j}^{i}(B):=\frac{\partial \sigma_{k+1}}{\partial B_{i}^{j}}(B)
$$

where $B=\left(B_{j}^{i}\right)$. If there is no confusion, we may omit the index $k$. We recall basic formulas about $\sigma_{k}$ and $T$.

$$
\begin{aligned}
\sigma_{k}(B) & =\frac{1}{k !} \delta_{j_{1} \cdots j_{k}}^{i_{1} \cdots i_{k}} B_{i_{1}}^{j_{1}} \cdots B_{i_{k}}^{j_{k}}=\frac{1}{k} \operatorname{tr}\left(T_{k-1}(B) B\right), \\
\left(T_{k}\right)_{j}^{i}(B) & =\frac{1}{k !} \delta_{j j_{1} \cdots j_{k}}^{i i_{1} \cdots i_{k}} B_{i_{1}}^{j_{1}} \cdots B_{i_{k}}^{j_{k}} .
\end{aligned}
$$

Let $(\Sigma, \gamma)$ be a hypersurface in $\mathbb{H}^{n}$ with $\gamma$ the induces metric and $B$ its second fundamental form. The $k$-th mean curvature of $\Sigma$ is defined

$$
\sigma_{k}=\sigma_{k}(B) \text {. }
$$

When $k=1, \sigma_{1}$ is just the ordinary mean curvature.

\section{A NEW MASS FOR ASYMPTOTICALLY HYPERBOLIC MANIFOLDS}

In this section, we will introduce a higher order mass for asymptotically hyperbolic manifolds which extends the mass defined in Subsection 2.1. Inspired by our recent work about a new mass on asymptotically flat manifolds [30], which was in turn motivated by the Gauss-Bonnet gravity [27, 28], we define it by using the Gauss-Bonnet curvature $L_{k}$. As indicated in Introduction, we need to modify the Gauss-Bonnet curvature $L_{k}$ a little bit in order to obtain a new one. More precisely, on a Riemannian manifold $\left(\mathcal{M}^{n}, g\right)$, we define a new four-tensor

$$
\widetilde{\operatorname{Riem}}_{i j s l}(g)=\widetilde{R}_{i j s l}(g):=R_{i j s l}(g)+g_{i s} g_{j l}-g_{i l} g_{j s} .
$$


Let us apply the same argument in Subsection 2.2 to define a new Gauss-Bonnet curvature with respect to this tensor $\overparen{\text { Riem }}$

$$
\widetilde{L}_{k}:=\frac{1}{2^{k}} \delta_{j_{1} j_{2} \cdots j_{2 k-1} j_{2 k}}^{i_{1} i_{2} \cdots i_{2 k-1} i_{2 k}} \widetilde{R}_{i_{1} i_{2}}^{j_{1} j_{2}} \cdots \widetilde{R}_{i_{2 k-1} i_{2 k}}^{j_{2 k-1} j_{2 k}}=\widetilde{R}_{s t j l} \widetilde{P}_{(k)}^{s t j l},
$$

where

$$
\widetilde{P}_{(k)}^{s t j l}:=\frac{1}{2^{k}} \delta_{j_{1} j_{2} \cdots j_{2 k-3} j_{2 k-2} j_{2 k-1} j_{2 k}}^{i_{1} i_{2} \cdots i_{2 k-3} i_{2 k-2} s t} \quad \widetilde{R}_{i_{1} i_{2}}^{j_{1} j_{2}} \cdots \widetilde{R}_{i_{2 k-3} i_{2 k-2}}^{j_{2 k-3} j_{2 k-2}} g^{j_{2 k-1} j} g^{j_{2 k} l} .
$$

It is easy to see that

$$
\widetilde{L}_{1}=L_{1}+n(n-1)=R+n(n-1)
$$

and

$$
\widetilde{L}_{2}=L_{2}+2(n-2)(n-3) R+n(n-1)(n-2)(n-3) .
$$

Moreover, we have

$$
\widetilde{P}_{(1)}^{s t j l}=P_{(1)}^{s t j l}=\frac{1}{2}\left(g^{s j} g^{t l}-g^{s l} g^{t j}\right),
$$

and

$$
\widetilde{P}_{(2)}^{s t j l}=\widetilde{R}^{s t j l}+\widetilde{R}^{t j} g^{s l}-\widetilde{R}^{s j} g^{t l}-\widetilde{R}^{t l} g^{s j}+\widetilde{R}^{s l} g^{t j}+\frac{1}{2} \widetilde{R}\left(g^{s j} g^{t l}-g^{s l} g^{t j}\right) .
$$

where

$$
\widetilde{R}^{s j}=g_{t l} \widetilde{R}^{s t j l}, \quad \widetilde{R}=g_{s j} \widetilde{R}^{s j} .
$$

By a direct calculation, one gets that

$$
\widetilde{P}_{(2)}^{s t j l}=P_{(2)}^{s t j l}+(n-2)(n-3) P_{(1)}^{s t j l},
$$

where $P_{2}$ and $P_{1}$ are defined in (2.15) and (2.13) respectively. Remark that $\widetilde{P}_{(k)}$ is a "good" linear combination of $P_{(i)}, i=1, \cdots, k$ with constant coefficients only depending on $n$ and $k$. It is clear that the tensor $\widetilde{P}_{(k)}$ satisfies the same properties as $P_{(k)}$, namely,

$$
\begin{gathered}
\widetilde{P}_{(k)}^{s t j l}=-\widetilde{P}_{(k)}^{t s j l}=-\widetilde{P}_{(k)}^{s t l j}=\widetilde{P}_{(k)}^{j l s t}, \\
\widetilde{P}_{(k)}^{s t j l}+\widetilde{P}_{(k)}^{t j s l}+\widetilde{P}_{(k)}^{j s t l}=0
\end{gathered}
$$

and

$$
\nabla_{s} \widetilde{P}_{(k)}^{s t j l}=0
$$

In the following, if there is no confusion, we just write $\widetilde{P}=\widetilde{P}_{(k)}$ for brief and denote the tensors associated with the hyperbolic metric $b$ with a bar. In view of (3.7) and (3.9), we observe that for asymptotically hyperbolic manifolds of decay order $\tau$, the Gauss-Bonnet curvature multiplying with a function $V \in\left\{\mathbb{N}_{b}\right\}$, i.e., $V \widetilde{L}_{k}$, can be expressed as a divergence term together with a term of faster decay.

First, since the difference of two Christoffel symbols is a tensor, we have the following formula

$$
\begin{aligned}
\Gamma_{i j}^{s}-\bar{\Gamma}_{i j}^{s} & =\frac{1}{2} g^{s l}\left(\bar{\nabla}_{i} g_{l j}+\bar{\nabla}_{j} g_{l i}-\bar{\nabla}_{l} g_{i j}\right) \\
& =\frac{1}{2} g^{s l}\left(\bar{\nabla}_{i} e_{l j}+\bar{\nabla}_{j} e_{l i}-\bar{\nabla}_{l} e_{i j}\right),
\end{aligned}
$$


where $e_{i j}:=g_{i j}-b_{i j}$ in local coordinates. And the difference of two curvature tensors in local coordinates is

$$
\begin{aligned}
R_{i j l}{ }^{t}{ }^{\prime}-\bar{R}_{i j l}{ }^{t} l= & \nabla_{i}\left(\Gamma_{j l}^{t}-\bar{\Gamma}_{j l}^{t}\right)-\nabla_{j}\left(\Gamma_{i l}^{t}-\bar{\Gamma}_{i l}^{t}\right) \\
& +\left(\Gamma_{i s}^{m}-\bar{\Gamma}_{i s}^{m}\right)\left(\Gamma_{j l}^{s}-\bar{\Gamma}_{j l}^{s}\right)-\left(\Gamma_{j s}^{m}-\bar{\Gamma}_{j s}^{m}\right)\left(\Gamma_{i l}^{s}-\bar{\Gamma}_{i l}^{s}\right) .
\end{aligned}
$$

We emphasize again that $\bar{\nabla}$ is the covariant derivative w.r.t to $b$ and $\nabla$ the covariant derivative w.r.t to $g$. By the divergence-free property of $\widetilde{P}$ and the fact that the quadratic terms of Christoffel symbols have a faster decay, we have from (3.7), (3.9) and (3.10) that

$$
\begin{aligned}
& V \widetilde{L}_{k}=V \widetilde{P}^{i j s l} \widetilde{R}_{i j s l}=V \widetilde{P}^{i j s l}\left(R_{i j s l}+g_{i s} g_{j l}-g_{i l} g_{j s}\right) \\
& =V \widetilde{P}^{i j s l}\left(g_{s t}\left(R_{i j}{ }^{t} l-\bar{R}_{i j}{ }^{t} l\right)+g_{i s} g_{j l}-g_{i l} g_{j s}+g_{s t} \bar{R}_{i j}{ }^{t} l\right) \\
& =V \widetilde{P}^{i j s l}\left(g_{s t}\left(R_{i j l}{ }^{t} l-\bar{R}_{i j}{ }^{t} l\right)+g_{i s}\left(g_{j l}-b_{j l}\right)-g_{j s}\left(g_{i l}-b_{i l}\right)\right) \\
& =2 V \widetilde{P}^{i j s l}\left(g_{s t} \nabla_{i}\left(\Gamma_{j l}^{t}-\bar{\Gamma}_{j l}^{t}\right)+g_{i s} e_{j l}\right)+O\left(e^{(-(k+1) \tau+1) r}\right) \\
& =V \nabla_{i}\left(\left(\bar{\nabla}_{l} e_{j s}+\bar{\nabla}_{j} e_{s l}-\bar{\nabla}_{s} e_{j l}\right) \widetilde{P}^{i j s l}\right)+2 V \widetilde{P}^{i j s l} b_{i s} e_{j l}+O\left(e^{(-(k+1) \tau+1) r}\right) \\
& =2 V \bar{\nabla}_{i}\left(\bar{\nabla}_{l} e_{j s} \widetilde{P}^{i j s l}\right)-2 V \widetilde{P}^{i j s l} b_{i l} e_{j s}+O\left(e^{(-(k+1) \tau+1) r}\right) \\
& =2 \bar{\nabla}_{i}\left(V\left(\bar{\nabla}_{l} e_{j s}\right) \widetilde{P}^{i j s l}\right)-2 \bar{\nabla}_{i} V\left(\bar{\nabla}_{l} e_{j s}\right) \widetilde{P}^{i j s l}-2 V \widetilde{P}^{i j s l} b_{i l} e_{j s}+O\left(e^{(-(k+1) \tau+1) r}\right) \\
& =2 \bar{\nabla}_{i}\left(V\left(\bar{\nabla}_{l} e_{j s}\right) \widetilde{P}^{i j s l}\right)-2 \bar{\nabla}_{l} V\left(\bar{\nabla}_{i} e_{j s}\right) \widetilde{P}^{i j s l}-2 V \widetilde{P}^{i j s l} b_{i l} e_{j s}+O\left(e^{(-(k+1) \tau+1) r}\right) \\
& (3.12)=2 \bar{\nabla}_{i}\left(\left(V \bar{\nabla}_{l} e_{j s}-e_{j s} \bar{\nabla}_{l} V\right) \widetilde{P}^{i j s l}\right)+2\left(\bar{\nabla}_{i} \bar{\nabla}_{l} V-V b_{i l}\right) e_{j s} \widetilde{P}^{i j s l}+O\left(e^{(-(k+1) \tau+1) r}\right) \text {. }
\end{aligned}
$$

Here we have used the fact for any tensor field $T$

$$
\bar{\nabla} T-\nabla T=O\left(e^{-\tau r}|T|\right),
$$

which follows from (3.10). Since $V \in \mathbb{N}_{b}$, which is defined in (2.2), the second term in (3.12) vanishes, and hence we conclude

$$
V \widetilde{L}_{k}=2 \bar{\nabla}_{i}\left(\left(V \bar{\nabla}_{l} e_{j s}-\bar{\nabla}_{l} V e_{j s}\right) \widetilde{P}^{i j s l}\right)+O\left(e^{(-(k+1) \tau+1) r}\right) .
$$

With this crucial expression, one can check that the limit

$$
H_{k}^{\Phi}(V)=\lim _{r \rightarrow \infty} \int_{S_{r}}\left(\left(V \bar{\nabla}_{l} e_{j s}-e_{j s} \bar{\nabla}_{l} V\right) \widetilde{P}_{(k)}^{i j s l}\right) \nu_{i} d \mu
$$

exists and is finite provided that $V \widetilde{L}_{k}$ is integrable and the decay order $\tau>\frac{n}{k+1}$. Here $\Phi$ is any diffeomorphism used in Definition 2.1. We remark that by (3.4), one can check that $2 H_{1}^{\Phi}(V)$ coincides with (2.5) defined by Chruściel and Herzlich [19]. Now we have

Theorem 3.1. Suppose $\left(\mathcal{M}^{n}, g\right)(2 k<n)$ is an asymptotically hyperbolic manifold of decay order $\tau>\frac{n}{k+1}$ and for $V \in \mathbb{N}_{b}$ defined in (2.2), $V \widetilde{L}_{k}$ is integrable in $\left(\mathcal{M}^{n}, g\right)$, then the mass functional $H_{k}^{\Phi}(V)$ defined in (3.15) is well-defined. 
Proof. This follows from (3.14) easily.

The above definition of an asymptotically hyperbolic mass functional involves the choice of coordinates at infinity. We need to show that it is a geometric invariant which does not depend on the choice of coordinates at infinity.

Theorem 3.2. Assume $\left(\mathcal{M}^{n}, g\right)$ is asymptotically hyperbolic manifold of decay order

$$
\tau>\frac{n}{k+1}
$$

and satisfies the integrable condition

$$
\int_{\mathcal{M}} V\left|\widetilde{L}_{k}\right|<\infty
$$

then $H_{k}^{\Phi}(V)$ does not depend on the choice of coordinates at infinity in the sense that if there are two maps $\Phi_{1}, \Phi_{2}$ satisfying (3.16), (3.17), then there exists an isometry $A$ of b, such that

$$
H_{k}^{\Phi_{2}}(V)=H_{k}^{\Phi_{1}}\left(V \circ A^{-1}\right) .
$$

Proof. The argument follows closely from the one given by Chruściel and Herzlich in the proof of hyperbolic mass [19]. See also [20, 40, 56]. The key point is to realize that when changing the coordinates at infinity, extra terms which do not decay fast enough to have vanishing integral can be collected in a divergence of some alternative 2-vector field.

First assume $\Phi_{1}$ and $\Phi_{2}$ are two coordinates at infinity satisfying (1.13) with $\tau>\frac{n}{k+1}$, then there exists an isometry $A$ of the background metric $b$ such that

$$
\Phi_{2}-\Phi_{1} \circ A=o\left(e^{-\frac{n}{k+1} r}\right) .
$$

Hence it suffices to prove

$$
H_{k}^{\Phi_{1} \circ A}(V)=H_{k}^{\Phi_{2}}(V) .
$$

As in [19] (see (2.17) there), we know that there is a well-defined vector field

$$
\zeta=o\left(e^{-\frac{n}{k+1} r}\right)
$$

such that

$$
\left(\Phi_{2}{ }^{-1}\right)^{*} g=\left(\Phi_{1}^{-1}\right)^{*} g+\mathscr{L}_{\zeta}\left(\Phi_{1}^{-1}\right)^{*} g+o\left(e^{-\frac{2 n}{k+1} r}\right)=\left(\Phi_{1}^{-1}\right)^{*} g+\mathscr{L}_{\zeta} b+o\left(e^{-\frac{2 n}{k+1} r}\right),
$$

where $\mathscr{L}$ is the Lie derivative.

For $p=1,2$, set

$$
\mathbb{U}_{p}^{i}=\left(V \bar{\nabla}_{l}\left(e_{p}\right)_{j s}-\left(e_{p}\right)_{j s} \bar{\nabla}_{l} V\right) \widetilde{P}^{i j s l}\left(g_{p}\right),
$$

where $g_{p}=\left(\Phi_{p}{ }^{-1}\right)^{*} g$, and $e_{p}=g_{p}-b$. It follows from (3.20) that

$$
\mathbb{U}_{2}^{i}-\mathbb{U}_{1}^{i}=\delta \mathbb{U}^{i}+o\left(e^{-(n-1) r}\right),
$$

where $\delta \mathbb{U}^{i}$ is a first-order term by linearizing $\mathbb{U}$ at $g_{1}$ and the remaining terms decay sufficiently fast so that they do not contribute when integrated at infinity. It remains to compute $\delta \mathbb{U}^{i}$. It is crucial to realize from (3.20) that

$$
\widetilde{P}^{i j s l}\left(g_{2}\right)-\widetilde{P}^{i j s l}\left(g_{1}\right)=o\left(e^{-\frac{k n}{k+1} r}\right) .
$$


When $k=1$ it is easy to prove, since $\widetilde{P}_{1}^{i j s l}(g)=\frac{1}{2}\left(g^{i s} g^{j l}-g^{i l} g^{j s}\right)$. For the general $k$, the proof of (3.21) is not obvious. We provide a detailed computation in Appendix B for the convenience of the reader. In the following computation, we write $\widetilde{P}=\widetilde{P}\left(g_{1}\right)$ for short. Hence we have

$$
\begin{aligned}
\delta \mathbb{U}^{i} & =\left(V \bar{\nabla}_{l}\left(\bar{\nabla}_{j} \zeta_{s}+\bar{\nabla}_{s} \zeta_{j}\right)-\left(\bar{\nabla}_{j} \zeta_{s}+\bar{\nabla}_{s} \zeta_{j}\right) \bar{\nabla}_{l} V\right) \widetilde{P}^{i j s l}+o\left(e^{-(n-1) r}\right) \\
& =\left(V \bar{\nabla}_{l} \bar{\nabla}_{j} \zeta_{s}+V \bar{\nabla}_{l} \bar{\nabla}_{s} \zeta_{j}-\bar{\nabla}_{j} \zeta_{s} \bar{\nabla}_{l} V+\bar{\nabla}_{l} \zeta_{j} \bar{\nabla}_{s} V\right) \widetilde{P}^{i j s l}+o\left(e^{-(n-1) r}\right) .
\end{aligned}
$$

Here in the second equality we have used the antisymmetry (3.7) to exchange the indices $s$ and $l$ in the fourth term. We apply the Ricci identity to deal with the second term in (3.22) as follows:

$$
\begin{aligned}
\left(\bar{\nabla}_{l} \bar{\nabla}_{s} \zeta_{j}-\bar{\nabla}_{s} \bar{\nabla}_{l} \zeta_{j}\right) \widetilde{P}^{i j s l} & =\left(\bar{R}_{l s j}{ }^{t} \zeta_{t}\right) \widetilde{P}^{i j s l} \\
& =-\left(b_{l j} \delta_{s}^{t}-\delta_{l}^{t} b_{s j}\right) \zeta_{t} \widetilde{P}^{i j s l} \\
& =-b_{l j} \zeta_{s} \widetilde{P}^{i j s l}+b_{s j} \zeta_{l} \widetilde{P}^{i j s l} \\
& =-2 b_{l j} \zeta_{s} \widetilde{P}^{i j s l},
\end{aligned}
$$

where in the last equality, (3.7) is used again. In view of (3.9), (3.22) and (3.23), we derive

$$
\begin{aligned}
\delta \mathbb{U}^{i}= & \left(V \bar{\nabla}_{l} \bar{\nabla}_{j} \zeta_{s}+V \bar{\nabla}_{s} \bar{\nabla}_{l} \zeta_{j}-2 b_{l j} \zeta_{s} V-\bar{\nabla}_{j} \zeta_{s} \bar{\nabla}_{l} V+\bar{\nabla}_{l} \zeta_{j} \bar{\nabla}_{s} V\right) \widetilde{P}^{i j s l}+o\left(e^{-(n-1) r}\right) \\
= & V \bar{\nabla}_{l} \bar{\nabla}_{j}\left(\zeta_{s} \widetilde{P}^{i j s l}\right)+V \bar{\nabla}_{s} \bar{\nabla}_{l}\left(\zeta_{j} \widetilde{P}^{i j s l}\right)-2 b_{l j} \zeta_{s} V \widetilde{P}^{i j s l}-\bar{\nabla}_{j}\left(\zeta_{s} \widetilde{P}^{i j s l}\right) \bar{\nabla}_{l} V \\
& +\bar{\nabla}_{l}\left(\zeta_{j} \widetilde{P}^{i j s l}\right) \bar{\nabla}_{s} V+o\left(e^{-(n-1) r}\right) \\
= & \bar{\nabla}_{l}\left(V \bar{\nabla}_{j}\left(\zeta_{s} \widetilde{P}^{i j s l}\right)\right)-2 \bar{\nabla}_{j}\left(\zeta_{s} \widetilde{P}^{i j s l}\right) \bar{\nabla}_{l} V-2 b_{l j} \zeta_{s} V \widetilde{P}^{i j s l}+\bar{\nabla}_{s}\left(V \bar{\nabla}_{l}\left(\zeta_{j} \widetilde{P}^{i j s l}\right)\right)+o\left(e^{-(n-1) r}\right) \\
= & \bar{\nabla}_{l}\left(V \bar{\nabla}_{j}\left(\zeta_{s} \widetilde{P}^{i j s l}\right)\right)-2 \bar{\nabla}_{j}\left(\zeta_{s} \widetilde{P}^{i j s l} \bar{\nabla}_{l} V\right)+2 \zeta_{s} \widetilde{P}^{i j s l} \bar{\nabla}_{j} \bar{\nabla}_{l} V-2 b_{l j} \zeta_{s} V \widetilde{P}^{i j s l} \\
& +\bar{\nabla}_{s}\left(V \bar{\nabla}_{l}\left(\zeta_{j} \widetilde{P}^{i j s l}\right)\right)+o\left(e^{-(n-1) r}\right) \\
= & \bar{\nabla}_{l}\left(V \bar{\nabla}_{j}\left(\zeta_{s} \widetilde{P}^{i j s l}\right)\right)-2 \bar{\nabla}_{j}\left(\zeta_{s} \widetilde{P}^{i j s l} \bar{\nabla}_{l} V\right)+\bar{\nabla}_{s}\left(V \bar{\nabla}_{l}\left(\zeta_{j} \widetilde{P}^{i j s l}\right)\right)+o\left(e^{-(n-1) r}\right) .
\end{aligned}
$$

In the last equality, we have used again $V \in \mathbb{N}_{b}$. By the first Bianchi identity (3.8) of $\widetilde{P}$, we have

$$
\bar{\nabla}_{l}\left(V \bar{\nabla}_{j}\left(\zeta_{s} \widetilde{P}^{i j s l}\right)\right)=-\bar{\nabla}_{l}\left(V \bar{\nabla}_{j}\left(\zeta_{s} \widetilde{P}^{j s i l}\right)\right)-\bar{\nabla}_{l}\left(V \bar{\nabla}_{j}\left(\zeta_{s} \widetilde{P}^{s i j l}\right)\right) .
$$

On the other hand, together with an exchange of a cycle $s, j, l$ and the symmetry, one has

$$
\bar{\nabla}_{l}\left(V \bar{\nabla}_{j}\left(\zeta_{s} \widetilde{P}^{s i j l}\right)\right)=\bar{\nabla}_{s}\left(V \bar{\nabla}_{l}\left(\zeta_{j} \widetilde{P}^{i j s l}\right)\right) .
$$

Hence we have

$$
\begin{aligned}
\delta \mathbb{U}^{i} & =-\bar{\nabla}_{l}\left(V \bar{\nabla}_{j}\left(\zeta_{s} \widetilde{P}^{j s i l}\right)\right)-2 \bar{\nabla}_{j}\left(\zeta_{s} \widetilde{P}^{i j s l} \bar{\nabla}_{l} V\right)+o\left(e^{-(n-1) r}\right) \\
& =-\bar{\nabla}_{l}\left(V \bar{\nabla}_{j}\left(\zeta_{s} \widetilde{P}^{j s i l}\right)+2 \zeta_{s} \widetilde{P}^{i l s j} \bar{\nabla}_{j} V\right)+o\left(e^{-(n-1) r}\right) \\
& =-\bar{\nabla}_{l} \alpha^{i l}+o\left(e^{-(n-1) r}\right),
\end{aligned}
$$

where

$$
\alpha^{i l}=V \bar{\nabla}_{j}\left(\zeta_{s} \widetilde{P}^{j s i l}\right)+2 \zeta_{s} \widetilde{P}^{i l s j} \bar{\nabla}_{j} V
$$

is anti-symmetric. Therefore we conclude that

$$
\mathbb{U}_{2}^{i}-\mathbb{U}_{1}^{i}=-\bar{\nabla}_{l} \alpha^{i l}+o\left(e^{-(n-1) r}\right),
$$


that is, up to the parts decay sufficiently fast which will not contribute to the integral at infinity, $\mathbb{U}_{2}^{i}-\mathbb{U}_{1}^{i}$ is a divergence of some alternative 2-vector field. We consider $\mathbb{U}_{2}-\mathbb{U}_{1}$ as a term of one form. Let $*$ be the Hodge operator with respect to the hyperbolic metric $b$. We have

$$
*\left(\mathbb{U}_{2}-\mathbb{U}_{1}\right)=d \widetilde{\alpha}+o\left(e^{-(n-1) r}\right),
$$

where $\widetilde{\alpha}$ is a $(n-2)$-form obtained from $\alpha$. This implies the geometric invariance of the mass functional (see also [19, 40, 56]).

We now give the precise definition of the Gauss-Bonnet-Chern mass (1.15) for asymptotically hyperbolic manifolds in the following definition.

Definition 3.3. If the mass functional $H_{k}^{\Phi}: \mathbb{N}_{b} \rightarrow \mathbb{R}$ is timelike future directed, i.e., $H_{k}^{\Phi}(V)>0$ for all $V \in \mathbb{N}^{+}$, then the higher order mass, the Gauss-Bonnet-Chern mass (1.15) for asymptotically hyperbolic manifolds, is defined by

$$
m_{k}^{\mathbb{H}}:=c(n, k) \inf _{V \in \mathbb{N}_{b}^{1}} H_{k}^{\Phi}(V) .
$$

Here $c(n, k)$ is the normalization constant given in (1.8).

The above results show that $m_{k}^{\mathbb{H}}$ is an invariant for asymptotically hyperbolic manifolds. A similar discussion as in subsection 2.1 implies

$$
m_{k}^{\mathbb{H}}=c(n, k) \inf _{\Phi} H_{k}^{\Phi}\left(V_{(0)}\right),
$$

where the infimum takes over all asymptotically hyperbolic coordinate $\Phi$ satisfying (3.16) and (3.17) and $V_{(0)}$ is a fixed element in $\mathbb{N}_{b}^{1}$. In the following we fix

$$
V=V_{(0)}=\cosh r \text {. }
$$

We end this section by a discussion on the range of $\tau$.

Remark 3.4. From (3.15), one can check directly that $m_{k}^{\mathbb{H}}$ vanishes if $\tau>\frac{n}{k}$, and hence the well-defined and the non-trivial range for the Gauss-Bonnet-Chern mass $m_{k}^{\mathbb{H}}$ is $\tau \in\left(\frac{n}{k+1}, \frac{n}{k}\right]$. The decay order of the anti-de Sitter Schwarzschild type metric (1.20) is just $\frac{n}{k}$. Its new mass equals to $m^{k}$. See Appendix A for more details.

\section{Positive mass theorem for asymptotically hyperbolic graphS}

In this section, we investigate a special case that asymptotically hyperbolic manifolds are given as graphs of asymptotically constant functions over hyperbolic space $\mathbb{H}^{n}$. For the new asymptotically hyperbolic mass, we can show that the corresponding Riemannian positive mass theorem holds for graphs when the modified Gauss-Bonnet curvature is nonnegative.

Following the notation in [22], we identify $\mathbb{H}^{n+1}$ with $\left(\mathbb{H}^{n} \times \mathbb{R}, b+V^{2} d s \otimes d s\right)$. Let $f: \mathbb{H}^{n} \rightarrow \mathbb{R}$ be a smooth function, then the induced metric on the graph

$$
\mathcal{M}:=\left\{(x, s) \in \mathbb{H}^{n} \times \mathbb{R} \mid f(x)=s\right\},
$$

is $\left(\mathcal{M}^{n}, g\right)=\left(\mathbb{H}^{n}, b+V^{2} d f \otimes d f\right)$. Suppose that the graph $(\mathcal{M}, g)$ is asymptotically hyperbolic of decay order $\tau>\frac{n}{k+1}$ and $V \widetilde{L}_{k}(g)$ is integrable. 
Remark 4.1. If $f: \mathbb{H}^{n} \rightarrow \mathbb{R}$ satisfies

$$
\|V \bar{\nabla} f\|_{b}+\left\|V \bar{\nabla}^{2} f\right\|_{b}+\left\|V \bar{\nabla}^{3} f\right\|_{b}=O\left(e^{-\frac{\tau}{2} r}\right), \quad \tau>\frac{n}{k+1},
$$

then the corresponding graph $\left(\mathcal{M}^{n}, g\right)=\left(\mathbb{H}^{n}, b+V^{2} d f \otimes d f\right)$ is asymptotically hyperbolic of decay order $\tau>\frac{n}{k+1}$.

In local coordinates,

$$
g_{i j}=b_{i j}+V^{2} \bar{\nabla}_{i} f \bar{\nabla}_{j} f
$$

and the inverse of $g_{i j}$ is

$$
g^{i j}=b^{i j}-\frac{V^{2} \bar{\nabla}^{i} f \bar{\nabla}^{j} f}{1+V^{2}|\bar{\nabla} f|^{2}},
$$

where the norm is taken with respect to the hyperbolic metric $b$.

Proposition 4.2. Suppose $\left(\mathcal{M}^{n}, g\right)=\left(\mathbb{H}^{n}, b+V^{2} \bar{\nabla} f \otimes \bar{\nabla} f\right)$, then we have

$$
\bar{\nabla}_{s}\left(\left(V \bar{\nabla}_{l} e_{t j}-e_{t j} \bar{\nabla}_{l} V\right) \widetilde{P}_{(k)}^{s t j l}\right)=\frac{V}{2} \widetilde{L}_{k}
$$

where $e_{i j}:=g_{i j}-b_{i j}=V^{2} \bar{\nabla}_{i} f \bar{\nabla}_{j} f$.

Proof. First note that the induced second fundamental form of $\mathcal{M}^{n}$ is given by (cf. [22])

$$
h_{i j}=\frac{V}{\sqrt{1+V^{2}|\bar{\nabla} f|^{2}}}\left(\bar{\nabla}_{i} \bar{\nabla}_{j} f+\frac{\bar{\nabla}_{i} f \bar{\nabla}_{j} V+\bar{\nabla}_{i} V \bar{\nabla}_{j} f}{V}+V\langle\bar{\nabla} f, \bar{\nabla} V\rangle \bar{\nabla}_{i} f \bar{\nabla}_{j} f\right) .
$$

Thus the shape operator is

$$
\begin{aligned}
h_{j}^{i} & :=g^{i s} h_{s j} \\
& =\frac{V}{\sqrt{1+V^{2}|\bar{\nabla} f|^{2}}}\left(\bar{\nabla}^{i} \bar{\nabla}_{j} f+\frac{\bar{\nabla}^{i} f \bar{\nabla}_{j} V}{V\left(1+V^{2}|\bar{\nabla} f|^{2}\right)}+\frac{\bar{\nabla}^{i} V \bar{\nabla}_{j} f}{V}-\frac{V^{2} \bar{\nabla}^{i} f \bar{\nabla}^{s} f \bar{\nabla}_{s} \bar{\nabla}_{j} f}{1+V^{2}|\bar{\nabla} f|^{2}}\right) .
\end{aligned}
$$

It follows from (3.10), (4.1) and (4.2) that

$$
\begin{aligned}
\Gamma_{i j}^{l}-\bar{\Gamma}_{i j}^{l}= & \frac{\bar{\nabla}^{l} f}{1+V^{2}|\bar{\nabla} f|^{2}}\left(V^{2} \bar{\nabla}_{i} \bar{\nabla}_{j} f+V \bar{\nabla}_{i} V \bar{\nabla}_{j} f+V \bar{\nabla}_{j} V \bar{\nabla}_{i} f\right) \\
& -V \bar{\nabla}_{i} f \bar{\nabla}_{j} f\left(\bar{\nabla}^{l} V-\frac{V^{2} \bar{\nabla}^{l} f\langle\bar{\nabla} f, \bar{\nabla} V\rangle}{1+V^{2}|\bar{\nabla} f|^{2}}\right) .
\end{aligned}
$$

In particular,

$$
\Gamma_{i s}^{s}-\bar{\Gamma}_{i s}^{s}=\frac{\bar{\nabla}^{s} f}{1+V^{2}|\bar{\nabla} f|^{2}}\left(V^{2} \bar{\nabla}_{i} \bar{\nabla}_{s} f+V \bar{\nabla}_{i} V \bar{\nabla}_{s} f\right)
$$

Recalling (3.1), by the Gauss formula we have

$$
\widetilde{R}_{i j}{ }^{s l}=h_{i}^{s} h_{j}^{l}-h_{i}^{l} h_{j}^{s} .
$$

Substituting (4.8) into (3.2), (3.3), we infer from (2.19) that

$$
\begin{aligned}
\widetilde{L}_{k} & =\delta_{j_{1} j_{2} \cdots j_{2 k-1} j_{2 k}}^{i_{1} i_{2} \cdots i_{2 k} i_{1}} h_{i_{1}}^{j_{1}} \cdots h_{i_{2 k}}^{j_{2 k}} \\
& =(2 k) ! \sigma_{2 k}(h)
\end{aligned}
$$


and

$$
\widetilde{P}_{(k)}^{s t j l}=\frac{1}{2} \delta_{j_{1} j_{2} \cdots j_{2 k-3} j_{2 k-2} j_{2 k-1} j_{2 k}}^{i_{1} i_{2} \cdots i_{1}} h_{i_{1}}^{j_{1}} h_{i_{2}}^{j_{2}} \cdots h_{i_{2 k-2}}^{j_{2 k-2}} g^{j_{2 k-1} j} g^{j_{2 k} l}
$$

which implies by (2.20) that

$$
2 \widetilde{P}_{(k)}^{s t j l} h_{s j}=(2 k-1) !\left(T_{(2 k-1)}\right)_{p}^{t} g^{p l}
$$

Here $h=\left(h_{i j}\right)$ is the second fundamental form given by (4.4). We derive from (3.7), (4.4) and (4.10) that

$$
\begin{aligned}
\left(V \bar{\nabla}_{l} e_{t j}-e_{t j} \bar{\nabla}_{l} V\right) \widetilde{P}_{(k)}^{s t j l} & =\left(V \bar{\nabla}_{l}\left(V^{2} \bar{\nabla}_{t} f \bar{\nabla}_{j} f\right)-V^{2} \bar{\nabla}_{t} f \bar{\nabla}_{j} f \bar{\nabla}_{l} V\right) \widetilde{P}_{(k)}^{s t j l} \\
& =\left(V^{3} \bar{\nabla}_{l} \bar{\nabla}_{t} f \bar{\nabla}_{j} f+V^{2} \bar{\nabla}_{l} V \bar{\nabla}_{t} f \bar{\nabla}_{j} f\right) \widetilde{P}_{(k)}^{s t j l} \\
& =V\left(\bar{\nabla}_{l} \bar{\nabla}_{t} f+\frac{\bar{\nabla}_{l} V \bar{\nabla}_{t} f}{V}\right) \widetilde{P}_{(k)}^{s t j l}\left(V^{2} \bar{\nabla}_{j} f\right) \\
& =h_{l t} \sqrt{1+V^{2}|\bar{\nabla} f|^{2}} \widetilde{P}_{(k)}^{s t j l}\left(V^{2} \bar{\nabla}_{j} f\right) \\
& =\frac{(2 k-1) !}{2}\left(T_{(2 k-1)}\right)_{p}^{s} g^{p j} V^{2} \bar{\nabla}_{j} f \sqrt{1+V^{2}|\bar{\nabla} f|^{2}} \\
& =\frac{(2 k-1) !}{2}\left(T_{(2 k-1)}\right)_{p}^{s} \frac{V^{2} \bar{\nabla}^{p} f}{\sqrt{1+V^{2}|\bar{\nabla} f|^{2}}},
\end{aligned}
$$

where the fourth equality follows from (4.4) and (3.7). Since the ambient space is the hyperbolic space $\mathbb{H}^{n}$ with constant curvature -1 , the Newton tensor is divergence-free with the induced metric $g$. (For the proof see [1] for instance.)

It follows that

$$
\begin{aligned}
& \left(\bar{\nabla}_{s}\left(T_{(2 k-1)}\right)_{p}^{s}\right) \bar{\nabla}^{p} f \\
= & \left(\nabla_{s}\left(T_{(2 k-1)}\right)_{p}^{s}\right) \bar{\nabla}^{p} f+\left(T_{(2 k-1)}\right)_{q}^{s}\left(\Gamma_{p s}^{q}-\bar{\Gamma}_{p s}^{q}\right) \bar{\nabla}^{p} f-\left(T_{(2 k-1)}\right)_{p}^{q}\left(\Gamma_{s q}^{s}-\bar{\Gamma}_{s q}^{s}\right) \bar{\nabla}^{p} f \\
= & \left(T_{(2 k-1)}\right)_{q}^{s}\left(\Gamma_{p s}^{q}-\bar{\Gamma}_{p s}^{q}\right) \bar{\nabla}^{p} f-\left(T_{(2 k-1)}\right)_{p}^{q}\left(\Gamma_{s q}^{s}-\bar{\Gamma}_{s q}^{s}\right) \bar{\nabla}^{p} f .
\end{aligned}
$$

Thus from (4.6) and (4.7), we have

$$
\begin{aligned}
\left(\bar{\nabla}_{s}\left(T_{(2 k-1)}\right)_{p}^{s}\right) \bar{\nabla}^{p} f= & \left(T_{(2 k-1)}\right)_{q}^{s}\left\{\frac{\bar{\nabla}^{q} f}{1+V^{2}|\bar{\nabla} f|^{2}}\left(V^{2} \bar{\nabla}_{p} \bar{\nabla}_{s} f+V \bar{\nabla}_{p} V \bar{\nabla}_{s} f+V \bar{\nabla}_{s} V \bar{\nabla}_{p} f\right)\right. \\
& \left.-V \bar{\nabla}_{p} f \bar{\nabla}_{s} f\left(\bar{\nabla}^{q} V-\frac{V^{2} \bar{\nabla}^{q} f\langle\bar{\nabla} f, \bar{\nabla} V\rangle}{1+V^{2}|\bar{\nabla} f|^{2}}\right)\right\} \bar{\nabla}^{p} f \\
& -\left(T_{(2 k-1)}\right)_{p}^{q} \frac{\bar{\nabla}^{s} f}{1+V^{2}|\bar{\nabla} f|^{2}}\left(V^{2} \bar{\nabla}_{q} \bar{\nabla}_{s} f+V \bar{\nabla}_{q} V \bar{\nabla}_{s} f\right) \bar{\nabla}^{p} f \\
= & \left(T_{(2 k-1)}\right)_{q}^{s}\left(V\langle\bar{\nabla} V, \bar{\nabla} f\rangle \bar{\nabla}^{q} f \bar{\nabla}_{s} f-V|\bar{\nabla} f|^{2} \bar{\nabla}_{s} f \bar{\nabla}^{q} V\right) .
\end{aligned}
$$


Therefore by above, we derive

$$
\begin{aligned}
& \bar{\nabla}_{s}\left(\left(T_{(2 k-1)}\right)_{p}^{s} \frac{V^{2} \bar{\nabla}^{p} f}{\sqrt{1+V^{2}|\bar{\nabla} f|^{2}}}\right) \\
= & \frac{V^{2}}{\sqrt{1+V^{2}|\bar{\nabla} f|^{2}}} \bar{\nabla}_{s}\left(T_{(2 k-1)}\right)_{p}^{s} \bar{\nabla}^{p} f+\left(T_{(2 k-1)}\right)_{p}^{s} \bar{\nabla}_{s}\left(\frac{V^{2} \bar{\nabla}^{p} f}{\sqrt{1+V^{2}|\bar{\nabla} f|^{2}}}\right) \\
= & \frac{V^{2}}{\sqrt{1+V^{2}|\bar{\nabla} f|^{2}}}\left(T_{(2 k-1)}\right)_{q}^{s}\left(V\langle\bar{\nabla} V, \bar{\nabla} f\rangle \bar{\nabla}^{q} f \bar{\nabla}_{s} f-V|\bar{\nabla} f|^{2} \bar{\nabla}_{s} f \bar{\nabla}^{q} V\right) \\
& +\left(T_{(2 k-1)}\right)_{p}^{s}\left(\frac{V^{2} \bar{\nabla}_{s} \bar{\nabla}^{p} f+2 V \bar{\nabla}_{s} V \bar{\nabla}^{p} f}{\sqrt{1+V^{2}|\bar{\nabla} f|^{2}}}-\frac{V^{2} \bar{\nabla}^{p} f\left(V|\bar{\nabla} f|^{2} \bar{\nabla}_{s} V+V^{2} \bar{\nabla}_{s} \bar{\nabla}^{q} f \bar{\nabla}_{q} f\right)}{\left(1+V^{2}|\bar{\nabla} f|^{2}\right)^{\frac{3}{2}}}\right) \\
= & \frac{V\left(T_{(2 k-1)}\right)_{p}^{s}}{\sqrt{1+V^{2}|\bar{\nabla} f|^{2}}}\left\{\left(V^{2}\langle\bar{\nabla} V, \bar{\nabla} f\rangle \bar{\nabla}^{p} f \bar{\nabla}_{s} f-V^{2}|\bar{\nabla} f|^{2} \bar{\nabla}_{s} f \bar{\nabla}^{p} V\right)\right. \\
& \left.+V \bar{\nabla}_{s} \bar{\nabla}^{p} f+\bar{\nabla}_{s} V \bar{\nabla}^{p} f+\frac{\bar{\nabla}_{s} V \bar{\nabla}^{p} f}{1+V^{2} \mid \bar{\nabla}^{2}}-\frac{V^{3} \bar{\nabla}^{p} f \bar{\nabla}_{s} \bar{\nabla}^{q} f \bar{\nabla}_{q} f}{1+V^{2}|\bar{\nabla} f|^{2}}\right\} \\
= & \frac{V\left(T_{(2 k-1)}\right)_{p}^{s}}{\sqrt{1+V^{2}|\bar{\nabla} f|^{2}}}\left(V^{2}\langle\bar{\nabla} V, \bar{\nabla} f\rangle \bar{\nabla}^{p} f \bar{\nabla}_{s} f-V^{2}|\bar{\nabla} f|^{2} \bar{\nabla}_{s} f \bar{\nabla}^{p} V+\bar{\nabla}_{s} V \bar{\nabla}^{p} f-\bar{\nabla}_{s} f \bar{\nabla}^{p} V\right) \\
& +V\left(T_{(2 k-1)}\right)_{p}^{s} h_{s}^{p} .
\end{aligned}
$$

We claim that the first term in the last equality of the above equation indeed vanishes. We define a new $(1,1)$ tensor by

$$
A_{s}^{p}:=V^{2}\langle\bar{\nabla} V, \bar{\nabla} f\rangle \bar{\nabla}^{p} f \bar{\nabla}_{s} f-V^{2}|\bar{\nabla} f|^{2} \bar{\nabla}_{s} f \bar{\nabla}^{p} V+\bar{\nabla}_{s} V \bar{\nabla}^{p} f-\bar{\nabla}_{s} f \bar{\nabla}^{p} V .
$$

Then we have

$$
\begin{aligned}
& \frac{V\left(T_{(2 k-1)}\right)_{p}^{s}}{\sqrt{1+V^{2}|\bar{\nabla} f|^{2}}}\left(V^{2}\langle\bar{\nabla} V, \bar{\nabla} f\rangle \bar{\nabla}^{p} f \bar{\nabla}_{s} f-V^{2}|\bar{\nabla} f|^{2} \bar{\nabla}_{s} f \bar{\nabla}^{p} V+\bar{\nabla}_{s} V \bar{\nabla}^{p} f-\bar{\nabla}_{s} f \bar{\nabla}^{p} V\right) \\
= & \frac{V\left(T_{(2 k-1)}\right)_{p}^{s}}{\sqrt{1+V^{2}|\bar{\nabla} f|^{2}}} A_{s}^{p}=\frac{V\left(T_{(2 k-1)}\right)^{s q}}{\sqrt{1+V^{2}|\bar{\nabla} f|^{2}}} g_{p q} A_{s}^{p} .
\end{aligned}
$$

We only need to show that $g_{p q} A_{s}^{p}$ is anti-symmetric, which is in fact easy to check

$$
\begin{aligned}
g_{p q} A_{s}^{p}= & \left(b_{p q}+V^{2} \bar{\nabla}_{p} f \bar{\nabla}_{q} f\right) A_{s}^{p} \\
= & V^{2}\langle\bar{\nabla} V, \bar{\nabla} f\rangle \bar{\nabla}_{q} f \bar{\nabla}_{s} f-V^{2}|\bar{\nabla} f|^{2} \bar{\nabla}_{s} f \bar{\nabla}_{q} V+\bar{\nabla}_{s} V \bar{\nabla}_{q} f-\bar{\nabla}_{s} f \bar{\nabla}_{q} V \\
& +V^{4}\langle\bar{\nabla} V, \bar{\nabla} f\rangle\left|\bar{\nabla}_{f}\right|^{2} \bar{\nabla}_{q} f \bar{\nabla}_{s} f-V^{4}|\bar{\nabla} f|^{2}\langle\bar{\nabla} f, \bar{\nabla} V\rangle \bar{\nabla}_{s} f \bar{\nabla}_{q} f \\
& +V^{2}|\bar{\nabla} f|^{2} \bar{\nabla}_{s} V \bar{\nabla}_{q} f-V^{2}\langle\bar{\nabla} f, \bar{\nabla} V\rangle \bar{\nabla}_{q} f \bar{\nabla}_{s} f \\
= & \left(1+V^{2}|\bar{\nabla} f|^{2}\right)\left(\bar{\nabla}_{s} V \bar{\nabla}_{q} f-\bar{\nabla}_{s} f \bar{\nabla}_{q} V\right) .
\end{aligned}
$$

Since $\left(T_{(2 k-1)}\right)^{s q}$ is symmetric, we get the desired result that

$$
\frac{V\left(T_{(2 k-1)}\right)_{p}^{s}}{\sqrt{1+V^{2}|\bar{\nabla} f|^{2}}}\left(V^{2}\langle\bar{\nabla} V, \bar{\nabla} f\rangle \bar{\nabla}^{p} f \bar{\nabla}_{s} f-V^{2}|\bar{\nabla} f|^{2} \bar{\nabla}_{s} f \bar{\nabla}^{p} V+\bar{\nabla}_{s} V \bar{\nabla}^{p} f-\bar{\nabla}_{s} f \bar{\nabla}^{p} V\right)=0
$$


which yields from (2.19) that

$$
\bar{\nabla}_{s}\left(\left(T_{(2 k-1)}\right)_{p}^{s} \frac{V^{2} \bar{\nabla}^{p} f}{\sqrt{1+V^{2}|\bar{\nabla} f|^{2}}}\right)=V\left(T_{(2 k-1)}\right)_{p}^{s} h_{s}^{p}=2 k V \sigma_{2 k}(h) .
$$

Finally, (4.3) follows from (4.9), (4.11) and (4.12).

Lemma 4.3. From (4.11), we have the following equivalent form of hyperbolic mass integral (3.15) for asymptotically hyperbolic graphs

$$
m_{k}^{\mathbb{H}}=c(n, k) \frac{(2 k-1) !}{2} \lim _{r \rightarrow \infty} \int_{S_{r}}\left(T_{(2 k-1)}\right)_{p}^{s} \frac{V^{2} \bar{\nabla}^{p} f}{\sqrt{1+V^{2}|\bar{\nabla} f|^{2}}} \nu_{s} d \mu .
$$

Remark 4.4. The method to show Proposition 4.2 is motivated by a classical paper of Reilly [60]. The method gives a simpler proof even for $k=1$ case (cf. [22]). A similar argument works for the Gauss-Bonnet-Chern mass for asymptotically flat manifolds, which provides a simply proof for the corresponding results in [30]. Moreover, it also provides a brief proof of the corresponding Penrose inequality. See the next Section.

Now we are ready to prove our main Theorem 1.3 .

Proof of Theorem 1.3. Applying divergence theorem with Proposition 4.2, we have

$$
\begin{aligned}
m_{k}^{\mathbb{H}} & =c(n, k) \int_{\mathcal{M}} \bar{\nabla}_{s}\left(\left(V \bar{\nabla}_{l} e_{t j}-e_{t j} \bar{\nabla}_{l} V\right) \widetilde{P}_{(k)}^{s t j l}\right) d V_{b} \\
& =\frac{c(n, k)}{2} \int_{\mathcal{M}} V \widetilde{L}_{k} d V_{b} \\
& =\frac{c(n, k)}{2} \int_{\mathcal{M}} \frac{V \widetilde{L}_{k}}{\sqrt{1+V^{2}|\bar{\nabla}|^{2}}} d V_{g}
\end{aligned}
$$

where the last equality holds due to the fact

$$
d V_{g}=\sqrt{\operatorname{det} g} d V_{b}=\sqrt{1+V^{2}|\bar{\nabla} f|^{2}} d V_{b} .
$$

\section{Penrose inequality for Graphs over $\mathbb{H}^{n}$ With A HORIZON Type Boundary}

Now we investigate the Penrose inequality related to the new mass for the asymptotically hyperbolic manifolds which can be realized as graphs. Let $\Omega$ be a bounded open set in $\mathbb{H}^{n}$ and $\Sigma=\partial \Omega$. If $f: \mathbb{H}^{n} \backslash \Omega \rightarrow \mathbb{R}$ is a smooth function such that each connected component of $\Sigma$ is in a level set of $f$ and

$$
|\bar{\nabla} f(x)| \rightarrow \infty \quad \text { as } x \rightarrow \Sigma,
$$

then the graph of $f,\left(\mathcal{M}^{n}, g\right)=\left(\mathbb{H}^{n} \backslash \Omega, b+V^{2} d f \otimes d f\right)$, is an asymptotically hyperbolic manifold with a horizon $\Sigma$. Without loss of generality we may assume that $\{(x, f(x)) \mid x \in \Sigma\}$ is included in $f^{-1}(0)$. In this case we can identify $\{(x, f(x)) \mid x \in \Sigma\}$ with $\Sigma$.

On $\Sigma$, the outer unit normal vector induced by the hyperbolic metric $b$ is

$$
\nu:=\nu^{i} \frac{\partial}{\partial x^{i}}=-\frac{\bar{\nabla} f}{|\bar{\nabla} f|} .
$$


Then

$$
\nu^{i}=-\frac{b^{i j} \bar{\nabla}_{j} f}{|\bar{\nabla} f|}=-\frac{\bar{\nabla}^{i} f}{|\bar{\nabla} f|} \quad \text { and } \quad \nu_{i}:=b_{i j} \nu^{j}=-\frac{\bar{\nabla}_{i} f}{|\bar{\nabla} f|} .
$$

Remark 5.1. One can easily check that the second fundamental forms of $\Sigma$ induced by $g$ and $b$ respectively differ by a multiple $\frac{1}{\sqrt{1+V^{2}|\bar{\nabla} f|^{2}}}$. Hence we have the following equivalent statements, provided $\Sigma \subset \mathbb{H}^{n}$ is strictly mean convex:

- $|\bar{\nabla} f|=\infty$ on $\Sigma$;

- $\Sigma$ is minimal, i.e., the induced mean curvature by the metric $g$ vanishes;

- $\Sigma$ is totally geodesic, i.e., the induced second fundamental form by the metric g vanishes.

Therefore, in this case $\Sigma$ is an area-minimizing horizon if and only if $|\bar{\nabla} f|=\infty$ on $\Sigma$. Hence $|\bar{\nabla} f|=\infty$ is a natural assumption for horizons.

Proof of Theorem 1.4. In view of (4.13), integrating by parts now gives an extra boundary term,

$$
\begin{aligned}
\frac{1}{c(n, k)} m_{k}^{\mathbb{H}} & =\frac{(2 k-1) !}{2} \lim _{r \rightarrow \infty} \int_{S_{r}}\left(T_{(2 k-1)}\right)_{p}^{s} \frac{V^{2} \bar{\nabla}^{p} f}{\sqrt{1+V^{2}|\bar{\nabla} f|^{2}}} \nu_{s} d \mu \\
& =\frac{1}{2} \int_{\mathbb{H}^{n} \backslash \Omega} \frac{V \widetilde{L}_{k}}{\sqrt{1+V^{2}|\bar{\nabla} f|^{2}}} d V_{g}-\frac{(2 k-1) !}{2} \int_{\Sigma}\left(T_{(2 k-1)}\right)_{p}^{s} \frac{V^{2} \bar{\nabla}^{p} f}{\sqrt{1+V^{2}|\bar{\nabla} f|^{2}}} \nu_{s} d \mu .
\end{aligned}
$$

We may choose the coordinates such that $\left\{\frac{\partial}{\partial x^{2}}, \cdots, \frac{\partial}{\partial x^{n}}\right\}$ span the tangential space of $\Sigma$ and $\frac{\partial}{\partial x^{1}}$ denotes the normal direction of $\Sigma$. To clarify the notations, in the following we will use the convention that the Latin letters stand for the index: $1,2, \cdots, n$ and the Greek letters stand for the index: $2, \cdots, n$. Due to the assumption that $\Sigma$ is in a level set of $f$, at any given point $p \in \Sigma$, we have

$$
\bar{\nabla}_{\alpha} f=0 \quad \text { and } \quad \bar{\nabla}_{\alpha} \bar{\nabla}_{\beta} f=|\bar{\nabla} f| B_{\alpha \beta},
$$

where $B$ is the second fundamental form with respect to the inward normal vector of $(\Sigma, \gamma)$ induced by the metric $b$. Therefore we infer from (2.19) and (2.20) that

$$
\begin{aligned}
\frac{1}{c(n, k)} m_{k}^{\mathbb{H}} & =\frac{1}{2} \int_{\mathbb{H}^{n} \backslash \Omega} \frac{V \widetilde{L}_{k}}{\sqrt{1+V^{2}|\bar{\nabla} f|^{2}}} d V_{g}+\frac{(2 k-1) !}{2} \int_{\Sigma}\left(T_{(2 k-1)}\right)_{1}^{1} \frac{V^{2}|\bar{\nabla} f|}{\sqrt{1+V^{2}|\bar{\nabla} f|^{2}}} d \mu \\
& =\frac{1}{2} \int_{\mathcal{M}^{n}} \frac{V \widetilde{L}_{k}}{\sqrt{1+V^{2}|\bar{\nabla} f|^{2}}} d V_{g}+\frac{(2 k-1) !}{2} \int_{\Sigma} \sigma_{2 k-1} V\left(\frac{V^{2}|\bar{\nabla} f|^{2}}{1+V^{2}|\bar{\nabla} f|^{2}}\right)^{k} d \mu \\
& =\frac{1}{2} \int_{\mathcal{M}^{n}} \frac{V \widetilde{L}_{k}}{\sqrt{1+V^{2}|\bar{\nabla} f|^{2}}} d V_{g}+\frac{(2 k-1) !}{2} \int_{\Sigma} V \sigma_{2 k-1} d \mu .
\end{aligned}
$$

Here we have used the simple fact in the second equality

$$
\left(T_{(2 k-1)}\right)_{1}^{1}=\frac{1}{(2 k-1) !} \delta_{1 i_{1} i_{2} \cdots j_{2 k-1}}^{1 j_{1} j_{2} \cdots j_{2 k-1}} h_{j_{1}}^{i_{1}} \cdots h_{j_{2 k-1}}^{i_{2 k-1}}=\left(\frac{V|\bar{\nabla} f|}{\sqrt{1+V^{2}|\bar{\nabla} f|^{2}}}\right)^{2 k-1} \sigma_{2 k-1}
$$

which follows from

$$
h_{\beta}^{\alpha}=\frac{V|\bar{\nabla} f|}{\sqrt{1+V^{2}|\bar{\nabla} f|^{2}}} b^{\alpha \delta} B_{\delta \beta}=\frac{V|\bar{\nabla} f|}{\sqrt{1+V^{2}|\bar{\nabla} f|^{2}}} B_{\beta}^{\alpha} \text {, }
$$


by (4.5) and (5.2) (where $B_{\beta}^{\alpha}:=\gamma^{\alpha \delta} B_{\delta \beta}=b^{\alpha \delta} B_{\delta \beta}$ ) and the last equality holds by the assumption (5.1). This finishes the proof of the Theorem.

Using the Alexandrov-Fenchel inequality (6.9) proved in the second part below, we have the following Penrose inequality, which is slightly stronger than Theorem 1.6.

Theorem 5.2 (Penrose Inequality). Let $\Omega$ be a bounded open set in $\mathbb{H}^{n}$ and $\Sigma=\partial \Omega$. If $f$ : $\mathbb{H}^{n} \backslash \Omega \rightarrow \mathbb{R}$ is a smooth function such that the graph $\left(\mathcal{M}^{n}, g\right)=\left(\mathbb{H}^{n} \backslash \Omega, b+V^{2} d f \otimes d f\right)$ is asymptotically hyperbolic of decay order $\tau>\frac{n}{k+1}$ and $V \widetilde{L}_{k}$ is integrable. Assume that each connected component of $\Sigma$ is in a level set of $f$ and $|\bar{\nabla} f(x)| \rightarrow \infty$ as $x \rightarrow \Sigma$. Let $\Omega_{i}$ be connected components of $\Omega, i=1, \cdots, l$ and let $\Sigma_{i}=\partial \Omega_{i}$ and suppose that each $\Omega_{i}$ is horospherical convex, then

$$
m_{k}^{\mathbb{H}} \geq c(n, k) \int_{\mathcal{M}^{n}} \frac{1}{2} \frac{V \widetilde{L}_{k}}{\sqrt{1+V^{2}|\bar{\nabla} f|^{2}}} d V_{g}+\sum_{i=1}^{l} \frac{1}{2^{k}}\left(\left(\frac{\left|\Sigma_{i}\right|}{\omega_{n-1}}\right)^{\frac{n}{k(n-1)}}+\left(\frac{\left|\Sigma_{i}\right|}{\omega_{n-1}}\right)^{\frac{n-2 k}{k(n-1)}}\right)^{k} .
$$

In particular, $\widetilde{L}_{k} \geq 0$ implies

$$
m_{k}^{\mathbb{H}} \geq \sum_{i=1}^{l} \frac{1}{2^{k}}\left(\left(\frac{\left|\Sigma_{i}\right|}{\omega_{n-1}}\right)^{\frac{n}{k(n-1)}}+\left(\frac{\left|\Sigma_{i}\right|}{\omega_{n-1}}\right)^{\frac{n-2 k}{k(n-1)}}\right)^{k}
$$

Moreover, equality is achieved by the anti-de Sitter Schwarzschild type metric (1.20).

Proof. The Penrose inequality follows from Theorem 1.4 and the Alexandrov-Fenchel inequality, Theorem 6.2 below. The last statement is proved in the following example.

We end this section with an interesting example.

Example 5.3. The generalized anti-de Sitter Schwarzschild space-time is given by

$$
-\left(1+\rho^{2}-\frac{2 m}{\rho^{\frac{n}{k}-2}}\right) d t^{2}+\left(1+\rho^{2}-\frac{2 m}{\rho^{\frac{n}{k}-2}}\right)^{-1} d \rho^{2}+\rho^{2} d \Theta^{2},
$$

where $d \Theta^{2}$ is the round metric on $\mathbb{S}^{n-1}$. When $k=1$ we recover anti-de Sitter Schwarzschild space-time of the Einstein gravity.

See also [21] or example 2.8 in [14]. Restricting to the time slice $t=0$, we obtain the anti-de Sitter Schwarzschild metric

$$
g_{\text {adS-Sch }}=\left(1+\rho^{2}-\frac{2 m}{\rho^{\frac{n}{k}-2}}\right)^{-1} d \rho^{2}+\rho^{2} d \Theta^{2} .
$$

One can realizes metric (5.4) as a graph over the hyperbolic metric $\mathbb{H}^{n}$. In the transformation of coordinates $\rho=\sinh r$, the hyperbolic metric $b=d r^{2}+\sinh ^{2} r d \Theta^{2}$ can be rewritten as

$$
b=\frac{d \rho^{2}}{1+\rho^{2}}+\rho^{2} d \Theta^{2} .
$$

Hence to explicitly express the metric (5.4) as a graph over $\mathbb{H}^{n}$, we need to find a function $f=f(\rho)$ satisfying

$$
V^{2}\left(\frac{\partial f}{\partial \rho}\right)^{2}=\frac{1}{1+\rho^{2}-\frac{2 m}{\rho^{\frac{n}{k}-2}}}-\frac{1}{1+\rho^{2}}
$$


Note that the function $\mathrm{V}$ is now equal to $\sqrt{1+\rho^{2}}$. Let $\rho_{0}$ be the solution of

$$
1+\rho^{2}-\frac{2 m}{\rho^{\frac{n}{k}-2}}=0 .
$$

Then when $\rho$ approaches $\rho_{0}$, we have $\frac{\partial f}{\partial \rho}=O\left(\left(\rho-\rho_{0}\right)^{-\frac{1}{2}}\right)$, so that we can solve

$$
f(\rho)=\int_{\rho_{0}}^{\rho} \frac{1}{\sqrt{1+s^{2}}} \sqrt{\frac{1}{1+s^{2}-\frac{2 m}{s^{\frac{n}{k}-2}}}-\frac{1}{1+s^{2}}} d s .
$$

One may compare with the Euclidean case where the Schwarzschild metric can be written as a graph over $\mathbb{R}^{n}$ [47]. This example is a generalization of the one considered in [22], where is in fact the case $k=1$.

One can check that the metric $g=g_{\text {adS-Sch }}$ satisfies

$$
\widetilde{L}_{k}(g)=0,
$$

and

$$
m_{k}^{\mathbb{H}}=m^{k} .
$$

For the convenience of the reader, we include the computations of (5.5) and (5.6) in Appendix A.

In this example, the horizon is given by the surface $\rho=\rho_{0}$ with $\rho_{0}$ being the solution of

$$
1+\rho^{2}-\frac{2 m}{\rho^{\frac{n}{k}-2}}=0 .
$$

Then the horizon is $\left\{S_{\rho_{0}}: \rho_{0}^{\frac{n}{k}}+\rho_{0}^{\frac{n}{k}-2}=2 m\right\}$ which implies the right hand side of inequality (1.19) is

$$
\begin{aligned}
R H S & =\frac{1}{2^{k}}\left(\left(\frac{\omega_{n-1} \rho_{0}^{n-1}}{\omega_{n-1}}\right)^{\frac{n}{k(n-1)}}+\left(\frac{\omega_{n-1} \rho_{0}^{n-1}}{\omega_{n-1}}\right)^{\frac{n-2 k}{k(n-1)}}\right)^{k} \\
& =\frac{1}{2^{k}}\left(\rho_{0}^{\frac{n}{k}}+\rho_{0}^{\frac{n}{k}-2}\right)^{k}=\frac{1}{2^{k}}(2 m)^{k} \\
& =m^{k}=m_{k}^{\mathbb{H}} .
\end{aligned}
$$

This means that equality in (1.19) is achieved by the adS Schwarzschild type metric (1.20).

\section{Appendix A. The Anti-De Sitter Schwarzschild type metric}

In this Appendix, we first compute the modified Gauss-Bonnet curvature $\widetilde{L}_{k}$ for the following Riemannian metric which was discussed in Section 5

$$
g_{\text {adS-Sch }}=\left(1+\rho^{2}-\frac{2 m}{\rho^{\frac{n}{k}-2}}\right)^{-1} d \rho^{2}+\rho^{2} d \Theta^{2} .
$$

Let us denote

$$
\varphi(\rho)=\sqrt{1+\rho^{2}-\frac{2 m}{\rho^{\frac{n}{k}-2}}} .
$$


We have for any vectors $X, Y$ tangential to the sphere $S_{\rho}$

$$
\begin{aligned}
\mathscr{R}(X \wedge \partial \rho)+X \wedge \partial \rho & =-\left(\frac{n}{2 k}-1\right) \frac{2 m}{\rho^{\frac{n}{k}}} X \wedge \partial \rho, \\
\mathscr{R}(X \wedge Y)+X \wedge Y & =\frac{2 m}{\rho^{\frac{n}{k}}} X \wedge Y,
\end{aligned}
$$

where $\mathscr{R}$ is the curvature operator. This follows from a directly computation. Inserting (A.2) and (A.3) into the (3.2), one can check that

$$
\widetilde{L}_{k}=(2 k) !\left(C_{n-1}^{2 k}-C_{n-1}^{2 k-1}\left(\frac{n}{2 k}-1\right)\right)\left(\frac{2 m}{\rho^{\frac{n}{k}}}\right)^{k}=0 .
$$

In the following, we compute the new mass of metric (A.1). Let $\frac{\partial}{\partial x^{1}}$ denotes the $\frac{\partial}{\partial \rho}$ direction and $\left\{\frac{\partial}{\partial x^{2}}, \cdots \frac{\partial}{\partial x^{n}}\right\}$ be an orthogonal basis on $\mathbb{S}^{n-1}$. We will use the convention that the Latin letters stand for the index: $1,2, \cdots, n$ and the Greek letters stand for the index: $2, \cdots, n$. Since the hyperbolic metric

$$
b=\frac{1}{1+\rho^{2}} d \rho^{2}+\rho^{2} d \Theta^{2},
$$

the only non-vanishing term is

$$
e_{11}=g_{11}-b_{11}=\frac{1}{1+\rho^{2}-\frac{2 m}{\rho^{\frac{n}{k}-2}}}-\frac{1}{1+\rho^{2}}=\frac{2 m}{\rho^{\frac{n}{k}+2}}+\text { lower order terms. }
$$

On the other hand, from (A.2) and (A.3), we know that for the curvature term $\widetilde{R}_{i_{1} i_{2}}{ }^{j_{1} j_{2}}$, the indices $\left\{j_{1}, j_{2}\right\}$ should be a permutation of $\left\{i_{1}, i_{2}\right\}$, otherwise $\widetilde{R}_{i_{1} i_{2}}{ }^{j_{1} j_{2}}$ vanishes. Consequently, by the definition of $\widetilde{P}_{(k)}^{s t l m}$ we compute by using (A.2), (A.3) that for fixed $\alpha, \beta$,

$$
\begin{aligned}
\widetilde{P}_{(k)}^{1 \alpha 1 \beta} & =\frac{1}{2^{k}} \delta_{j_{1} j_{2} \cdots j_{2 k-3} j_{2 k-2} j_{2 k-1} j_{2 k}}^{i_{1} i_{2} \cdots i_{2 k-3} i_{2 k-2} 1 \alpha} \widetilde{R}_{i_{1} i_{2}}^{j_{1} j_{2}} \cdots \widetilde{R}_{i_{2 k-3} i_{2 k-2}}^{j_{2 k-3} j_{2 k-2}} g^{j_{2 k-1} 1} g^{j_{2 k} \beta} \\
& =\frac{1}{2^{k}} \delta_{j_{1} j_{2} \cdots j_{2 k-3} j_{2 k-2} 1 \alpha}^{i_{1} i_{2} \cdots i_{2 k-3} i_{2 k-2} 1 \alpha} \widetilde{R}_{i_{1} i_{2}}^{j_{1} j_{2}} \cdots \widetilde{R}_{i_{2 k-3} i_{2 k-2}}^{j_{2 k-3} j_{2 k-2}} g^{11} g^{\alpha \beta} \\
& =\frac{1}{2^{k}} 2^{k-1}(2 k-2) ! C_{n-2}^{2 k-2}\left(\frac{2 m}{\rho^{\frac{n}{k}}}\right)^{k-1} g^{11} g^{\alpha \beta} \\
& =2^{k-2}(2 k-2) ! C_{n-2}^{2 k-2}\left(\frac{m}{\rho^{\frac{n}{k}}}\right)^{k-1}\left(1+\rho^{2}-\frac{2 m}{\rho^{\frac{n}{k}-2}}\right) g^{\alpha \beta} .
\end{aligned}
$$

Moreover, from above calculations, one can check that this kind of term $\widetilde{P}_{(k)}^{1 \alpha \beta \delta}=0$. And a direct computation gives

$$
\bar{\nabla}_{t}\left(e_{\alpha \beta}\right)=0
$$

Noticing the fact

we derive from above that

$$
\nu_{\alpha}=0
$$

$$
\left(V \bar{\nabla}_{t}\left(e_{j s}\right)-e_{j s} \bar{\nabla}_{t} V\right) \widetilde{P}_{(k)}^{i j s t} \nu_{i}=V \bar{\nabla}_{\beta}\left(e_{1 \alpha}\right) \widetilde{P}_{(k)}^{1 \alpha 1 \beta} \nu_{1} .
$$

Using

$$
\bar{\Gamma}_{\alpha \beta}^{1}=-\rho\left(1+\rho^{2}\right) \delta_{\alpha \beta},
$$


and recalling $V=\sqrt{1+\rho^{2}}$, we compute further from above that

$$
\left(V \bar{\nabla}_{t}\left(e_{j s}\right)-e_{j s} \bar{\nabla}_{t} V\right) \widetilde{P}_{(k)}^{i j s t} \nu_{i}=\frac{2 m}{\rho^{\frac{n}{k}-2}} \delta_{\alpha \beta} \widetilde{P}_{(k)}^{1 \alpha 1 \beta} \nu_{1}+\text { lower order terms. }
$$

Hence we infer from (A.4) and (A.5) that

$$
\begin{aligned}
\left(V \bar{\nabla}_{t}\left(e_{j s}\right)-e_{j s} \bar{\nabla}_{t} V\right) \widetilde{P}_{(k)}^{i j s t} \nu_{i} & =2^{k-1}(2 k-2) ! C_{n-2}^{2 k-2} \frac{m^{k}}{\rho^{n-2}}(n-1) \nu_{1}+\text { lower order terms } \\
& =2^{k-1}(2 k-2) ! C_{n-2}^{2 k-2} \frac{m^{k}}{\rho^{n-1}}(n-1)+\text { lower order terms }
\end{aligned}
$$

where in the second equality we have used the fact $\nu_{1}=\frac{1}{\sqrt{1+\rho^{2}}}$. Therefore we conclude

$$
\begin{aligned}
m_{k}^{\mathbb{H}} & =\frac{(n-2 k) !}{2^{k-1}(n-1) ! \omega_{n-1}} \lim _{\rho \rightarrow \infty} \int_{S_{\rho}}\left(V \bar{\nabla}_{t}\left(e_{j s}\right)-e_{j s} \bar{\nabla}_{t} V\right) \widetilde{P}_{(k)}^{i j s t} \nu_{i} d \mu \\
& =\frac{(n-2 k) !}{2^{k-1}(n-1) ! \omega_{n-1}} 2^{k-1}(2 k-2) ! C_{n-2}^{2 k-2} \frac{m^{k}}{\rho^{n-1}}(n-1) \rho^{n-1} \omega_{n-1} \\
& =m^{k}
\end{aligned}
$$

\section{Appendix B. Proof of 3.21}

In this Appendix, we give the proof of (3.21).

Proof of (3.21). Denote $g_{1}=\left(\Phi_{1}^{-1}\right)^{*} g$ and $g_{2}=\left(\Phi_{2}^{-1}\right)^{*} g$. Recall from (3.20) that we have

$$
g_{2}-g_{1}=\mathcal{L}_{\zeta} b+o\left(e^{-\frac{2 n}{k+1} r}\right), \quad \text { with } \zeta=o\left(e^{-\frac{n}{k+1} r}\right) .
$$

It follows easily that

$$
\widetilde{R}_{i j}{ }^{s p}\left(g_{1}\right)=o\left(e^{-\frac{n}{k+1} r}\right), \quad \widetilde{R}_{i j}{ }^{s p}\left(g_{2}\right)=o\left(e^{-\frac{n}{k+1} r}\right) .
$$

In order to prove (3.21), in view of (B.1) and (3.3), it suffices to show that

$$
\widetilde{R}_{i j}{ }^{s p}\left(g_{2}\right)-\widetilde{R}_{i j}{ }^{s p}\left(g_{1}\right)=o\left(e^{-\frac{2 n}{k+1} r}\right) .
$$

In the proof, what we need to take care of is why the linear terms involving $\zeta$ cancel.

First by (3.1), we compute

$$
\begin{aligned}
& \widetilde{R}_{i j}{ }^{s p}\left(g_{2}\right)-\widetilde{R}_{i j}{ }^{s p}\left(g_{1}\right) \\
= & \left(R_{i j}{ }^{s p}\left(g_{2}\right)+\delta_{i}^{s} \delta_{j}^{p}-\delta_{i}^{p} \delta_{j}^{s}\right)-\left(R_{i j}{ }^{s p}\left(g_{1}\right)+\delta_{i}^{s} \delta_{j}^{p}-\delta_{i}^{p} \delta_{j}^{s}\right) \\
= & g_{2}^{p q} R_{i j}{ }^{s}{ }_{q}\left(g_{2}\right)-g_{1}^{p q} R_{i j}{ }^{s}{ }_{q}\left(g_{1}\right)=\left(g_{2}^{p q}-g_{1}^{p q}\right) R_{i j}{ }^{s}{ }_{q}\left(g_{2}\right)+g_{1}^{p q}\left(R_{i j}{ }^{s}{ }_{q}\left(g_{2}\right)-R_{i j}{ }^{s}{ }_{q}\left(g_{1}\right)\right) \\
= & -\left(\mathcal{L}_{\zeta} b\right)^{p q}\left(-\left(\delta_{i}^{s}\left(g_{2}\right)_{j q}-\delta_{j}^{s}\left(g_{2}\right)_{i q}\right)\right)+g_{1}^{p q}\left(R_{i j}{ }^{s}{ }_{q}\left(g_{2}\right)-R_{i j}{ }^{s}{ }_{q}\left(g_{1}\right)\right)+o\left(e^{-\frac{2 n}{k+1} r}\right) \\
=: & I+I I+o\left(e^{-\frac{2 n}{k+1} r}\right),
\end{aligned}
$$

where in the third equality, we have used the fact (B.2) and expression (B.1). 
We begin with the simpler term $I$. We have from (B.1) that

$$
\begin{aligned}
I & :=-\left(\mathcal{L}_{\zeta} b\right)^{p q}\left(-\left(\delta_{i}^{s}\left(g_{2}\right)_{j q}-\delta_{j}^{s}\left(g_{2}\right)_{i q}\right)\right) \\
& =-\left(\bar{\nabla}^{p} \zeta^{q}+\bar{\nabla}^{q} \zeta^{p}\right)\left(-\left(\delta_{i}^{s} b_{j q}-\delta_{j}^{s} b_{i q}\right)\right)+o\left(e^{-\frac{2 n}{k+1} r}\right) \\
& =\delta_{i}^{s}\left(\bar{\nabla}^{p} \zeta_{j}+\bar{\nabla}_{j} \zeta^{p}\right)-\delta_{j}^{s}\left(\bar{\nabla}^{p} \zeta_{i}+\bar{\nabla}_{i} \zeta^{p}\right)+o\left(e^{-\frac{2 n}{k+1} r}\right) .
\end{aligned}
$$

In the following, we deal with the second term $I I$. In view of (3.10), we have

$$
\begin{aligned}
\Gamma_{i j}^{s}\left(g_{2}\right)-\Gamma_{i j}^{s}\left(g_{1}\right) & =\frac{1}{2}\left(g_{2}\right)^{s l}\left(\nabla_{i}^{g_{1}}\left(g_{2}-g_{1}\right)_{l j}+\nabla_{j}^{g_{1}}\left(g_{2}-g_{1}\right)_{l i}-\nabla_{l}^{g_{1}}\left(g_{2}-g_{1}\right)_{i j}\right) \\
& =\frac{1}{2} b^{s l}\left(\bar{\nabla}_{i}\left(g_{2}-g_{1}\right)_{l j}+\bar{\nabla}_{j}\left(g_{2}-g_{1}\right)_{l i}-\bar{\nabla}_{l}\left(g_{2}-g_{1}\right)_{i j}\right)+o\left(e^{-\frac{2 n}{k+1} r}\right) .
\end{aligned}
$$

Substituting above into (3.11) and noting that the quadratic terms of Christoffel symbols having faster decay, we calculate

$$
\begin{aligned}
R_{i j}{ }_{q}\left(g_{2}\right)-R_{i j}{ }_{q}\left(g_{1}\right)= & \bar{\nabla}_{i}\left(\Gamma_{j q}^{s}\left(g_{2}\right)-\Gamma_{j q}^{s}\left(g_{1}\right)\right)-\bar{\nabla}_{j}\left(\Gamma_{i q}^{s}\left(g_{2}\right)-\Gamma_{i q}^{s}\left(g_{1}\right)\right)+o\left(e^{-\frac{2 n}{k+1} r}\right) \\
= & \frac{1}{2} b^{s l}\left(\bar{\nabla}_{i} \bar{\nabla}_{j}\left(g_{2}-g_{1}\right)_{l q}+\bar{\nabla}_{i} \bar{\nabla}_{q}\left(g_{2}-g_{1}\right)_{l j}-\bar{\nabla}_{i} \bar{\nabla}_{l}\left(g_{2}-g_{1}\right)_{j q}\right. \\
& \left.-\bar{\nabla}_{j} \bar{\nabla}_{i}\left(g_{2}-g_{1}\right)_{l q}-\bar{\nabla}_{j} \bar{\nabla}_{q}\left(g_{2}-g_{1}\right)_{l i}+\bar{\nabla}_{j} \bar{\nabla}_{l}\left(g_{2}-g_{1}\right)_{i q}\right)+o\left(e^{-\frac{2 n}{k+1} r}\right) \\
= & \frac{1}{2} b^{s l}\left[\left(\bar{\nabla}_{i} \bar{\nabla}_{j}\left(g_{2}-g_{1}\right)_{l q}-\bar{\nabla}_{j} \bar{\nabla}_{i}\left(g_{2}-g_{1}\right)_{l q}\right)+\left(\bar{\nabla}_{i} \bar{\nabla}_{q} \bar{\nabla}_{j} \zeta_{l}-\bar{\nabla}_{j} \bar{\nabla}_{q} \bar{\nabla}_{i} \zeta_{l}\right)\right. \\
& +\left(\bar{\nabla}_{i} \bar{\nabla}_{q} \bar{\nabla}_{l} \zeta_{j}-\bar{\nabla}_{i} \bar{\nabla}_{l} \bar{\nabla}_{q} \zeta_{j}\right)+\left(\bar{\nabla}_{j} \bar{\nabla}_{l} \bar{\nabla}_{i} \zeta_{q}-\bar{\nabla}_{i} \bar{\nabla}_{l} \bar{\nabla}_{j} \zeta_{q}\right) \\
& \left.+\left(\bar{\nabla}_{j} \bar{\nabla}_{l} \bar{\nabla}_{q} \zeta_{i}-\bar{\nabla}_{j} \bar{\nabla}_{q} \bar{\nabla}_{l} \zeta_{i}\right)\right]+o\left(e^{-\frac{2 n}{k+1} r}\right) \\
= & \frac{1}{2} b^{s l}\left(I I_{1}+I I_{2}+I I_{3}+I I_{4}+I I_{5}\right)+o\left(e^{-\frac{2 n}{k+1} r}\right),
\end{aligned}
$$

where in the third equality, we have used (B.1) and rearranged these terms.

In the following computations, we mainly rely on the Ricci identity to calculate each term carefully. Applying the Ricci identity, we have

$$
\begin{aligned}
I I_{1} & :=\bar{\nabla}_{i} \bar{\nabla}_{j}\left(g_{2}-g_{1}\right)_{l q}-\bar{\nabla}_{j} \bar{\nabla}_{i}\left(g_{2}-g_{1}\right)_{l q} \\
& =\left(g_{2}-g_{1}\right)_{l t}\left(-\bar{R}_{i j}{ }^{t} q\right)+\left(g_{2}-g_{1}\right)_{t q}\left(-\bar{R}_{i j}{ }^{t}{ }_{l}\right) \\
& =\left(g_{2}-g_{1}\right)_{l t}\left(\delta_{i}^{t} b_{j q}-\delta_{j}^{t} b_{i q}\right)+\left(g_{2}-g_{1}\right)_{t q}\left(\delta_{i}^{t} b_{j l}-\delta_{j}^{t} b_{i l}\right) \\
& =\left(g_{2}-g_{1}\right)_{i l} b_{j q}-\left(g_{2}-g_{1}\right)_{l j} b_{i q}+\left(g_{2}-g_{1}\right)_{i q} b_{j l}-\left(g_{2}-g_{1}\right)_{j q} b_{i l} .
\end{aligned}
$$

Using the Ricci identity three times, we obtain,

$$
\begin{aligned}
I I_{2} & :=\bar{\nabla}_{i} \bar{\nabla}_{q} \bar{\nabla}_{j} \zeta_{l}-\bar{\nabla}_{j} \bar{\nabla}_{q} \bar{\nabla}_{i} \zeta_{l} \\
& =\left(\bar{\nabla}_{i} \bar{\nabla}_{q} \bar{\nabla}_{j} \zeta_{l}-\bar{\nabla}_{q} \bar{\nabla}_{i} \bar{\nabla}_{j} \zeta_{l}\right)+\left(\bar{\nabla}_{q} \bar{\nabla}_{i} \bar{\nabla}_{j} \zeta_{l}-\bar{\nabla}_{q} \bar{\nabla}_{j} \bar{\nabla}_{i} \zeta_{l}\right)+\left(\bar{\nabla}_{q} \bar{\nabla}_{j} \bar{\nabla}_{i} \zeta_{l}-\bar{\nabla}_{j} \bar{\nabla}_{q} \bar{\nabla}_{i} \zeta_{l}\right) \\
& =\bar{\nabla}_{j} \zeta_{t}\left(-\bar{R}_{i q}{ }^{t}\right)+\bar{\nabla}_{t} \zeta_{l}\left(-\bar{R}_{i q}{ }^{t}{ }_{j}\right)+\bar{\nabla}_{q} \zeta_{t}\left(-\bar{R}_{i j}{ }^{t}{ }_{l}\right)+\bar{\nabla}_{t} \zeta_{l}\left(-\bar{R}_{q j}{ }^{t}{ }_{i}\right)+\bar{\nabla}_{i} \zeta_{t}\left(-\bar{R}_{q j}{ }^{t} l\right) \\
& =b_{q l}\left(\bar{\nabla}_{j} \zeta_{i}-\bar{\nabla}_{i} \zeta_{j}\right)-b_{i l}\left(\bar{\nabla}_{q} \zeta_{j}+\bar{\nabla}_{j} \zeta_{q}\right)+b_{j l}\left(\bar{\nabla}_{q} \zeta_{i}+\bar{\nabla}_{i} \zeta_{q}\right)+b_{q j} \bar{\nabla}_{i} \zeta_{l}-b_{q i} \bar{\nabla}_{j} \zeta_{l} .
\end{aligned}
$$


Similarly, we get

$$
\begin{aligned}
I I_{3} & :=\bar{\nabla}_{i} \bar{\nabla}_{q} \bar{\nabla}_{l} \zeta_{j}-\bar{\nabla}_{i} \bar{\nabla}_{l} \bar{\nabla}_{q} \zeta_{j}=-b_{q j} \bar{\nabla}_{i} \zeta_{l}+b_{l j} \bar{\nabla}_{i} \zeta_{q}, \\
I I_{4} & :=\bar{\nabla}_{j} \bar{\nabla}_{l} \bar{\nabla}_{i} \zeta_{q}-\bar{\nabla}_{i} \bar{\nabla}_{l} \bar{\nabla}_{j} \zeta_{q} \\
& =b_{q l}\left(\bar{\nabla}_{i} \zeta_{j}-\bar{\nabla}_{j} \zeta_{i}\right)-b_{j q}\left(\bar{\nabla}_{l} \zeta_{i}+\bar{\nabla}_{i} \zeta_{l}\right)+b_{i q}\left(\bar{\nabla}_{l} \zeta_{j}+\bar{\nabla}_{j} \zeta_{l}\right)+b_{l i} \bar{\nabla}_{j} \zeta_{q}-b_{l j} \bar{\nabla}_{i} \zeta_{q}, \\
I I_{5} & :=\bar{\nabla}_{j} \bar{\nabla}_{l} \bar{\nabla}_{q} \zeta_{i}-\bar{\nabla}_{j} \bar{\nabla}_{q} \bar{\nabla}_{l} \zeta_{i}=-b_{l i} \bar{\nabla}_{j} \zeta_{q}+b_{q i} \bar{\nabla}_{j} \zeta_{l} .
\end{aligned}
$$

By a simplification, we infer by (B.1) that

$$
\begin{aligned}
& I I_{1}+\left(I I_{2}+I I_{3}+I I_{4}+I I_{5}\right) \\
= & \left(g_{2}-g_{1}\right)_{i l} b_{j q}-\left(g_{2}-g_{1}\right)_{l j} b_{i q}+\left(g_{2}-g_{1}\right)_{i q} b_{j l}-\left(g_{2}-g_{1}\right)_{j q} b_{i l} \\
& -b_{j q}\left(\bar{\nabla}_{i} \zeta_{l}+\bar{\nabla}_{l} \zeta_{i}\right)+b_{j l}\left(\bar{\nabla}_{q} \zeta_{i}+\bar{\nabla}_{i} \zeta_{q}\right)-b_{i l}\left(\bar{\nabla}_{j} \zeta_{q}+\bar{\nabla}_{q} \zeta_{j}\right)+b_{i q}\left(\bar{\nabla}_{l} \zeta_{j}+\bar{\nabla}_{j} \zeta_{l}\right) \\
= & -2 b_{i l}\left(\bar{\nabla}_{j} \zeta_{q}+\bar{\nabla}_{q} \zeta_{j}\right)+2 b_{j l}\left(\bar{\nabla}_{i} \zeta_{q}+\bar{\nabla}_{q} \zeta_{i}\right)+o\left(e^{-\frac{2 n}{k+1} r}\right) .
\end{aligned}
$$

Hence we conclude

$$
\begin{aligned}
I I & :=g_{1}^{p q}\left(R_{i j}^{s}{ }_{q}\left(g_{2}\right)-R_{i j}{ }^{s}{ }_{q}\left(g_{1}\right)\right) \\
& =g_{1}^{p q}\left(\frac{1}{2} b^{s l}\right)\left(-2 b_{i l}\left(\bar{\nabla}_{j} \zeta_{q}+\bar{\nabla}_{q} \zeta_{j}\right)+2 b_{j l}\left(\bar{\nabla}_{i} \zeta_{q}+\bar{\nabla}_{q} \zeta_{i}\right)\right)+o\left(e^{-\frac{2 n}{k+1} r}\right) \\
& =b^{p q}\left(\frac{1}{2} b^{s l}\right)\left(-2 b_{i l}\left(\bar{\nabla}_{j} \zeta_{q}+\bar{\nabla}_{q} \zeta_{j}\right)+2 b_{j l}\left(\bar{\nabla}_{i} \zeta_{q}+\bar{\nabla}_{q} \zeta_{i}\right)\right)+o\left(e^{-\frac{2 n}{k+1} r}\right) \\
& =-\delta_{i}^{s}\left(\bar{\nabla}_{j} \zeta^{p}+\bar{\nabla}^{p} \zeta_{j}\right)+\delta_{j}^{s}\left(\bar{\nabla}_{i} \zeta^{p}+\bar{\nabla}^{p} \zeta_{i}\right)+o\left(e^{-\frac{2 n}{k+1} r}\right) .
\end{aligned}
$$

Finally, the desired result (B.3) follows from (B.4) and (B.5).

\section{Part 2. Alexandrov-Fenchel inequalites in $\mathbb{H}^{n}$}

\section{INTRODUCTION}

The second part of this paper is about weighted Alexandrov-Fenchel inequalities in $\mathbb{H}^{n}$, which is used to prove the Penrose inequality for asymptotically hyperbolic graphs in the last Section. This part has its own and independent interest. For the convenience of the reader we give an introduction on the Alexandrov-Fenchel inequalities.

The classical isoperimetric inequality and its generalization, the Alexandrov-Fenchel inequalities, play an important role in integral geometry, convex geometry and differential geometry. Let $\Omega$ be a smooth bounded domain in $\mathbb{R}^{n}$ with boundary $\Sigma$. The classical isoperimetric inequality is

$$
\operatorname{Area}(\Sigma) \geq n^{\frac{n-1}{n}} \omega_{n-1}^{\frac{1}{n}} \operatorname{Vol}(\Omega)^{\frac{n-1}{n}} .
$$

Equality holds if and only if $\Omega$ is a geodesic ball. When $n=2$, (6.1) is

$$
L^{2} \geq 4 \pi A,
$$

where $L$ is the length of a curve $\zeta$ in $\mathbb{R}^{2}$ and $A$ is the area of the enclosed domain by $\zeta$. The Alexandrov-Fenchel inequalities (in fact, its special class) are

$$
\int_{\Sigma} \sigma_{k} d \mu \geq C_{n-1}^{k} \omega_{n-1}\left(\frac{1}{C_{n-1}^{j}} \frac{1}{\omega_{n-1}} \int_{\Sigma} \sigma_{j} d \mu\right)^{\frac{n-1-k}{n-1-j}}, \quad 0 \leq j<k \leq n-1,
$$


for any convex hypersurface $\Sigma$. These inequalities are optimal, in the sense that equality holds if and only if $\Sigma$ is a geodesic sphere. The Alexandrov-Fenchel inequalities have been also extended to certain class of non-convex hypersurfaces. See [15, 36, 44] for instance.

It is natural to ask if the isoperimetric inequality and the Alexandrov-Fenchel inequalities hold in the hyperbolic space. The motivations to study this problem come from integral geometry and also from the recent study of the Penrose inequality for various mass. The classical isoperimetric problem between volume and area was solved by Schmidt 64 70 years ago. When $n=2$ the corresponding isoperimetric inequality is

$$
L^{2} \geq 4 \pi A+A^{2}
$$

where $L$ is the length of a curve $\zeta$ in $\mathbb{H}^{2}$ and $A$ is the area of the enclosed domain by $\zeta$. However, unlike the Euclidean space, for general $n$ there is no such explicit form.

There are many attempts to establish Alexandrov-Fenchel type inequalities in the hyperbolic space $\mathbb{H}^{n}$. See, for example, [6, 29, 62]. In [29], Gallego-Solanes proved by using integral geometry the following interesting inequality for convex domains in $\mathbb{H}^{n}$,

$$
\int_{\Sigma} \sigma_{k} d \mu>c C_{n-1}^{k}|\Sigma|
$$

where $c=1$ if $k>1$ and $|\Sigma|$ is the area of $\Sigma$. Here $d \mu$ is the area element of the induced metric $\gamma$ from the hyperbolic space and $\sigma_{k}$ is defined in (2.21). The above inequality (6.4) $(k>1)$ is sharp in the sense that the constant $c$ could not be improved. However, this inequality is far away from being optimal, especially when $|\Sigma|$ is small. One may compare it with the optimal inequalities given below.

There are two classes of the Alexandrov-Fenchel inequalities: One is without a weight $V$ and another with a weight $V$. The weight $V$ is an element in $\mathbb{N}_{b}^{1}$ considered in Part I. Here as before we fix it

$$
V=\cosh r
$$

in $\mathbb{H}^{n}=\mathbb{R}^{+} \times \mathbb{S}^{n-1}$ with the hyperbolic metric $b=d r^{2}+\sinh ^{2} r g_{\mathbb{S}^{n-1}}$. Here $r$ is the hyperbolic distance to a fixed point $x_{0}$.

The Alexandrov-Fenchel inequalities without weight are closely related to integral geometry in $\mathbb{H}^{n}$.

Theorem 6.1 ([51, 32, 33, 73]). Let $1 \leq k \leq n-1$. Any horospherical convex hypersurface $\Sigma$ in $\mathbb{H}^{n}$ satisfies

$$
\int_{\Sigma} \sigma_{k} d \mu \geq C_{n-1}^{k} \omega_{n-1}\left\{\left(\frac{|\Sigma|}{\omega_{n-1}}\right)^{\frac{2}{k}}+\left(\frac{|\Sigma|}{\omega_{n-1}}\right)^{\frac{2}{k} \frac{(n-k-1)}{n-1}}\right\}^{\frac{k}{2}} .
$$

Equality holds if and only if $\Sigma$ is a geodesic sphere.

Inequality (6.5) was called as a hyperbolic Alexandrov-Fenchel inequality in [32. (6.5) was proved in [51] for $k=2$ under a weaker condition that $\Sigma$ is star-shaped and 2-convex, in [32] for $k=4$ and in [33] for general even $k$. For $k=1$, (6.5) was proved in [33] with a help of a result of Cheng and $\mathrm{Xu}$ [16]. For general odd integer $k$, inequality (6.5) was proved very recently in [73]. Inequality (6.5) with odd $k$ will be used in this paper.

The proof of inequality (6.5) with even $k$ in [51, 32, 33] uses various inverse curvature flows studied by Gerhardt [34]. One of the crucial step is to show the monotonicity of a geometric 
integral under some inverse curvature flow. This geometric integral is in fact the integral of the Gauss-Bonnet curvature $L_{k}(g)$ of the induced metric $g$ on the embedded hypersurface $\Sigma \subset \mathbb{H}^{n}$

$$
\int_{\Sigma} L_{k}(g) d \mu(g)
$$

Hence, by using this method one in fact obtains an optimal Sobolev type inequality for (6.6), which then implies the Alexandrov-Fenchel inequality (6.5). The proof of inequality (6.5) in [73. works for all $k$. In the proof, a quermassintegral preserving curvature flow was used. The quermassintegral will also be used in this paper. For its definition see at the end of this Section. Theorem 6.1 was also proved by Guan-Li under a condition that the hypersurface is star-shaped, together with a technical condition, by using a modified inverse curvature flow [37.

The Alexandrov-Fenchel inequalities with weight for $k=1$ was studied in [12] and [26], where they were called Minkowski type inequalities. Motivated by the study of the quasi-local mass and the Penrose inequality, Brendle-Hung-Wang [12] established the following Minkowski type inequalities (i.e., $k=1$ )

$$
\int_{\Sigma}\left(V \sigma_{1}-(n-1)\langle\bar{\nabla} V, \nu\rangle\right) d \mu \geq(n-1) \omega_{n-1}^{\frac{1}{n-1}}|\Sigma|^{\frac{n-2}{n-1}}
$$

and de Lima and Girão [26] proved the following related inequality

$$
\int_{\Sigma} V \sigma_{1} d \mu \geq(n-1) \omega_{n-1}\left(\left(\frac{|\Sigma|}{\omega_{n-1}}\right)^{\frac{n-2}{n-1}}+\left(\frac{|\Sigma|}{\omega_{n-1}}\right)^{\frac{n}{n-1}}\right),
$$

if $\Sigma$ is star-shaped and mean convex (i.e. $\sigma_{1}>0$ ). The method to prove (6.7) is still the use of an inverse curvature flow and also works for anti-de Sitter Schwarzschild manifolds. Moreover this method motivates the work of [51] and [32, 33. Inequality (6.8) was mentioned in Part I.

It is natural to ask if general weighted Alexandrov-Fenchel inequalities hold. In this part of the paper, we give an affirmative answer, at least for horospherical convex hypersurfaces.

Theorem 6.2. Let $\Sigma$ be a horospherical convex hypersurface in the hyperbolic space $\mathbb{H}^{n}$. We have

$$
\int_{\Sigma} V p_{2 k+1} d \mu \geq \omega_{n-1}\left(\left(\frac{|\Sigma|}{\omega_{n-1}}\right)^{\frac{n}{(k+1)(n-1)}}+\left(\frac{|\Sigma|}{\omega_{n-1}}\right)^{\frac{n-2 k-2}{(k+1)(n-1)}}\right)^{k+1} .
$$

Equality holds if and only if $\Sigma$ is a centered geodesic sphere in $\mathbb{H}^{n}$.

By a centered geodesic sphere in $\mathbb{H}^{n}$ we mean $\left\{r=r_{0}\right\}$, a geodesic sphere centered at the fixed point $x_{0}$, for some constant $r_{0}>0$. Here and in the following, for the simplicity of notation we denote by

$$
p_{j}=\frac{1}{C_{n-1}^{j}} \sigma_{j}
$$

the normalized $j$-th mean curvature.

For the proof of this Theorem, we first need several refined Minkowski integral formulas, which will be given in Section 7. (In fact, unlike the usual Minowski identity (7.4) below, what we have are inequalities. In order to distinguish with the Minkowski type inequalities obtained in [12] 
and [26], we call them Minkowski integral formulas.) A crucial point is to show the following inequality

$$
E(\Sigma):=\int_{\Sigma} V p_{k+1} d \mu-\int_{\Sigma}\left(V p_{k-1}+\frac{p_{k+1}}{V}\right) d \mu \geq 0
$$

To show this inequality we use the following "conformal flow"

$$
\frac{d}{d t} \Sigma(t)=-V \nu
$$

which was used first by Brendle 11] to prove his generalized Heintze-Karcher inequality. With the Minkowski integral formulas given in Section 7 below, we prove that $E$ is non-increasing along this conformal flow. Using the monotonicity of the quermassintegral we prove that $E(\Sigma(t))$ tends to 0 , when hypersurfaces $\Sigma(t)$ shrink to a point along the conformal flow. Therefore we have $E(\Sigma) \geq 0$ for any horospherical convex hypersurface. Inequality (6.11) enables us to use an iteration argument as follows. When $k=0$, (6.9) is just (6.8), which was proved in [26]. Assume that (6.9) holds for $k-1$, we then use (6.11) and Theorem 6.1 to show that (6.9) holds for $k$.

It is an interesting question if Theorem 6.2 holds under the weaker condition that the hypersurface is convex, or even that the hypersurface is just so-called $k$-convex. In our proof of Theorem 6.2, the horospherical condition is used just in the proof of Theorem 8.2, which we believe is unnecessary, and in the use of Theorem 6.1. For Theorem 6.1 there are evidence that the convexity should be enough in [37.

We end this section by recalling the definition of the quermassintegrals [63, 65, 71]. For a (geodesically) convex domain $K \subset \mathbb{H}^{n}$ with boundary $\partial K=\Sigma$, the quermassintegrals are defined by

$$
W_{k}(K):=\frac{(n-k) \omega_{k-1} \cdots \omega_{0}}{n \omega_{n-2} \cdots \omega_{n-k-1}} \int_{\mathcal{L}_{k}} \chi\left(L_{k} \cap K\right) d L_{k}, \quad k=1, \cdots, n-1 ;
$$

where $\mathcal{L}_{k}$ is the space of $k$-dimensional totally geodesic subspaces $L_{k}$ in $\mathbb{H}^{n}$ and $d L_{k}$ is the natural (invariant) measure on $\mathcal{L}_{k}$. The function $\chi$ is given by $\chi(K)=1$ if $K \neq \emptyset$ and $\chi(\emptyset)=0$. For simplicity, we also use the convention

$$
W_{0}(K)=\operatorname{Vol}(K), \quad W_{n}(K)=\frac{\omega_{n-1}}{n} .
$$

Remark that by definition we know

$$
W_{1}(K)=\frac{1}{n}|\partial K|
$$

From integral geometry we know that the quermassintegrals and the curvature integrals in $\mathbb{H}^{n}$ do not coincide. Nevertheless they are closely related (see e.g. [71], Proposition 7):

$$
\int_{\Sigma} p_{k} d \mu=n\left(W_{k+1}(K)+\frac{k}{n-k+1} W_{k-1}(K)\right), \quad k=1, \cdots, n-1,
$$

\section{Minkowski integral FORMUlas}

Let $u=\langle\bar{\nabla} V, \nu\rangle>0$ be the support function, where $\nu$ is the outer normal vector. Here and in the following, we denote the connections on $\mathbb{H}^{n}$ and $\Sigma$ by $\bar{\nabla}$ and $\nabla$ respectively. In the following, for a hypersurface $\left(\Sigma^{n-1}, g\right)$ embedded in the hyperbolic space $\mathbb{H}^{n}$, we denote the second fundamental form by $h_{i j}$ and the shape operator $h_{i}^{j}:=h_{i k} g^{j k}$. The $k$-th Newton 
transformation is defined in (2.18). Before stating the main results, let us collect some basic facts with the weight.

Lemma 7.1. (1) The gradient vector field $\bar{\nabla} V$ of the weight function $V$ is a conformal vector field, i.e.,

$$
\bar{\nabla}_{X} \bar{\nabla} V=V X
$$

for any vector field $X$.

(2) We have the following Minkowski identity with weight $V$

$$
\nabla_{j}\left(T_{k}^{i j} \nabla_{i} V\right)=-(k+1) u \sigma_{k+1}+(n-(k+1)) \sigma_{k} V .
$$

(3) There is a relation between the weight $V$ and the support function $u$

$$
V^{2}=1+u^{2}+|\nabla V|^{2} .
$$

Proof. (17.1) is well-known. For (7.2), one can calculate it directly with the help of (7.1) or see (8.4) in [1]. To prove (7.3), first note that the orthogonal composition

$$
\bar{\nabla} V=\nabla V+u \nu
$$

implies

$$
|\bar{\nabla} V|^{2}=|\nabla V|^{2}+u^{2}
$$

Recalling $V=\cosh r$ and the simple fact $|\bar{\nabla} r|=1$, we have

$$
V^{2}-1=\sinh ^{2} r=|\bar{\nabla} V|^{2} .
$$

Thus we complete the proof.

In view of (6.10), it follows from (7.2) that we have the following well-known Minkowski integral formula between $p_{k}$ and $p_{k+1}$,

$$
\int_{\Sigma} u p_{k+1} d \mu=\int_{\Sigma} V p_{k} d \mu
$$

This is the classical Minkowski integral identity in $\mathbb{H}^{n}$.

In order to prove our optimal inequalities, we need to generalize the Minkowski type identity between $p_{k}$ and $p_{k+1}$, which are now only inequalities. See (7.6), (7.8), Proposition 7.4 and (8.5) below. To distinguish between such inequalities and the Minkowski type inequality (see (6.7) and (6.8)), we call them Minkowski integral formulas, between integrals involving $\sigma_{k}$ and $\sigma_{k+1}$.

Proposition 7.2. Let $\Sigma$ be a convex hypersurface in the hyperbolic space $\mathbb{H}^{n}$ and for any integer $1 \leq k \leq n-1$. We have

$$
\int_{\Sigma} u V p_{k} d \mu=\int_{\Sigma} V^{2} p_{k-1} d \mu+\frac{1}{C_{n-1}^{k}} \int_{\Sigma} \frac{1}{k}\left(T_{k-1}\right)^{i j} \nabla_{i} V \nabla_{j} V d \mu .
$$

Moreover, we have

$$
\int_{\Sigma} u V p_{k} d \mu \geq \int_{\Sigma} V^{2} p_{k-1} d \mu
$$

Equality holds if and only if $\Sigma$ is a centered geodesic sphere in $\mathbb{H}^{n}$. 
Proof. In view of (7.2), we have

$$
\frac{1}{k C_{n-1}^{k}} \nabla_{j}\left(T_{k-1}^{i j} \nabla_{i} V\right)=-u p_{k}+p_{k-1} V
$$

Multiplying the above equation by the function $V$ and integrating by parts, we obtain the desired result (7.5). The convexity of $\Sigma$ implies that $\left(T_{k-1}\right)^{i j}$ is positively definite (for the proof see [35] for instance), namely,

$$
\left(T_{k-1}\right)^{i j} \nabla_{i} V \nabla_{j} V \geq 0
$$

Hence (7.6) holds. When equality in (7.6) holds, we have $\nabla V=0$ which implies that $\Sigma$ is a centered geodesic sphere in $\mathbb{H}^{n}$.

Proposition 7.3. Let $\Sigma$ be a convex hypersurface in the hyperbolic space $\mathbb{H}^{n}$ and for any integer $1 \leq k \leq n-1$. We have

$$
\int_{\Sigma}\left(u^{2} p_{k}-u V p_{k-1}\right) d \mu=\frac{1}{k C_{n-1}^{k}} \int_{\Sigma}\left(T_{k-1}\right)^{i j} \nabla_{i} V \nabla_{j} u d \mu \geq 0
$$

Equality holds if and only if $\Sigma$ is a centered geodesic sphere in $\mathbb{H}^{n}$.

Proof. Multiplying (7.7) by the support function $u$ and integrating by parts, we have

$$
\int_{\Sigma}\left(u^{2} p_{k}-u V p_{k-1}\right) d \mu=\int_{\Sigma} \frac{1}{k C_{n-1}^{k}}\left(T_{k-1}\right)^{i j} \nabla_{i} V \nabla_{j} u d \mu .
$$

Next we compute

$$
\nabla_{i} u=\bar{\nabla}_{i} u=\left\langle\bar{\nabla}_{i} \bar{\nabla} V, \nu\right\rangle+\left\langle\bar{\nabla} V, \bar{\nabla}_{i} \nu\right\rangle=\left\langle\nabla V, \nabla_{i} \nu\right\rangle=\nabla_{l} V h_{i}^{l},
$$

where $h_{i j}$ is the second fundamental form of $\Sigma$ in $\mathbb{H}^{n}$. Here we have used the fact (7.1) that the vector filed $\bar{\nabla} V$ is conformal, and $\bar{\nabla}_{i} \nu$ has only tangential part and thus the tangential part of $\bar{\nabla} V$ is $\nabla V$. Going back into (7.9), we obtain

$$
\int_{\Sigma}\left(u^{2} p_{k} d \mu-u V p_{k-1}\right) d \mu=\frac{1}{C_{n-1}^{k}} \int_{\Sigma} \frac{1}{k}\left(T_{k-1}\right)^{i j} h_{j}^{l} \nabla_{l} V \nabla_{i} V d \mu .
$$

We note that $\left(T_{k-1}\right)^{i j}$ and $h^{l i}$ are both positive-definite and the multiplication is commutative. Thus, by a simple fact of linear algebra, we know that the product of matrices $\left(T_{k-1}\right)^{i j} h_{i}^{l}$ is still positive-definite, and hence

$$
\int_{\Sigma}\left(T_{k-1}\right)^{i j} h_{j}^{l} \nabla_{l} V \nabla_{i} V d \mu \geq 0
$$

As a consequence, we have

$$
\int_{\Sigma} u^{2} p_{k} d \mu \geq \int_{\Sigma} u V p_{k-1} d \mu
$$

When equality holds, we have $\nabla V \equiv 0$. Hence, $\Sigma$ is a centered geodesic sphere.

For the later use, we need the following inequalities.

Proposition 7.4. Let $\Sigma$ be a convex hypersurface in the hyperbolic space $\mathbb{H}^{n}$ and for any integer $1 \leq k \leq n-2$. We have

$$
\int_{\Sigma} V^{2} p_{k+1} d \mu \geq \int_{\Sigma} V^{2} p_{k-1} d \mu+\int_{\Sigma} p_{k+1} d \mu
$$


and

$$
\int_{\Sigma} u V p_{k+1} d \mu \geq \int_{\Sigma} u V p_{k-1} d \mu+\int_{\Sigma} \frac{u}{V} p_{k+1} d \mu .
$$

Equality in the above inequalities holds if and only if $\Sigma$ is a centered geodesic sphere in $\mathbb{H}^{n}$.

Proof. Applying (7.3), we obtain

$$
\int_{\Sigma} V^{2} p_{k+1} d \mu=\int_{\Sigma} u^{2} p_{k+1} d \mu+\int_{\Sigma} p_{k+1} d \mu+\int_{\Sigma}|\nabla V|^{2} p_{k+1} d \mu
$$

From Proposition 7.2 and Proposition 7.3 .

$$
\int_{\Sigma} u^{2} p_{k+1} d \mu \geq \int_{\Sigma} u V p_{k} d \mu \geq \int_{\Sigma} V^{2} p_{k-1} d \mu
$$

Therefore, the desired inequality (7.11) follows. To prove (7.12), using (7.3) and Proposition 7.2, Proposition 7.3 again, we have

$$
\int_{\Sigma} u V p_{k+1} d \mu \geq \int_{\Sigma} V^{2} p_{k}=\int_{\Sigma} p_{k} d \mu+\int_{\Sigma} u^{2} p_{k} d \mu+\int_{\Sigma}|\nabla V|^{2} p_{k} d \mu \geq \int_{\Sigma} p_{k} d \mu+\int_{\Sigma} u V p_{k-1} d \mu .
$$

Multiplying (7.7) by the function $\frac{1}{V}$ and integrating by parts, we have

$$
\begin{aligned}
\int_{\Sigma} p_{k} d \mu & =\int_{\Sigma} \frac{u}{V} p_{k+1} d \mu+\frac{1}{(k+1) C_{n-1}^{k+1}} \int_{\Sigma} \frac{1}{V^{2}}\left(T_{k}\right)^{i j} \nabla_{i} V \nabla_{j} V d \mu \\
& \geq \int_{\Sigma} \frac{u}{V} p_{k+1} d \mu .
\end{aligned}
$$

Here in the last inequality, we have used the fact that $\left(T_{k}\right)^{i j}$ is positive-definite. Hence we prove (7.12). The equality cases follow readily.

\section{A crucial Minkowski integral formula}

In this section, we consider the following functional

$$
E:=\int_{\Sigma}\left(V p_{k+1}-V p_{k-1}-\frac{p_{k+1}}{V}\right) d \mu
$$

Before discussing further, let us recall some basic facts of the general evolution equations. Precisely, consider a one-parameter family $X(t,):. \Sigma^{n-1} \rightarrow \mathbb{H}^{n}, t \in[0, \epsilon)$ of closed, isometrically embedded hypersurfaces evolving by

$$
\frac{\partial X}{\partial t}=F \nu
$$

where $\nu$ is the outward unit normal to $\Sigma_{t}=X(t,$.$) and F$ is a general speed function. For the convenience of the reader, we collect some evolution formulas in the following lemma.

Lemma 8.1. Along flow (8.2), we have

(1) $\frac{\partial}{\partial t} d \mu=F \sigma_{1} d \mu$

(2) $\frac{\partial V}{\partial t}=u F$,

(3) $\frac{\partial \sigma_{k}}{\partial t}=-T_{k-1}^{i j} \nabla_{i} \nabla_{j} F-F\left(\sigma_{1} \sigma_{k}-(k+1) \sigma_{k+1}\right)+(n-k) F \sigma_{k-1}$, 
(4) $\frac{\partial}{\partial t} \int_{\Sigma} V p_{k} d \mu=\int_{\Sigma}\left((k+1) u p_{k}+(n-k-1) V p_{k+1}\right) F d \mu$,

(5) For $l \geq 0$, we have

$$
\begin{aligned}
\frac{\partial}{\partial t} \int_{\Sigma} \frac{p_{k}}{V^{l}} d \mu= & -l(k+1) \int_{\Sigma} \frac{p_{k}}{V^{l+1}} u F d \mu-\frac{l(l+1)}{C_{n-1}^{k}} \int_{\Sigma} \frac{\left(\nabla_{i} V\right)\left(\nabla_{j} V\right)}{V^{l+2}} T_{k-1}^{i j} F d \mu \\
& +k(l+1) \int_{\Sigma} \frac{p_{k-1}}{V^{l}} F d \mu+\int_{\Sigma}(n-k-1) \frac{p_{k+1}}{V^{l}} F d \mu .
\end{aligned}
$$

In particular, under flow $F=-V$ we have the following simple form

$$
\frac{\partial}{\partial t} \int_{\Sigma} \frac{p_{k}}{V^{l}} d \mu=\int_{\Sigma} \frac{(l-k) u p_{k}-(n-k-1) V p_{k+1}}{V^{l}} d \mu .
$$

Proof. (1) and (2) are included in [43], and (3) follows from [61. Here we provide a proof for (4). In view of (7.2), we have along flow (8.2) that

$$
\begin{aligned}
\frac{\partial}{\partial t} \int_{\Sigma} V \sigma_{k} d \mu & =\int_{\Sigma} \frac{\partial V}{\partial t} \sigma_{k} d \mu+\int_{\Sigma} V \frac{\partial \sigma_{k}}{\partial t} d \mu+\int_{\Sigma} V \sigma_{k}\left(F \sigma_{1}\right) d \mu \\
& =\int_{\Sigma}\left\{u F \sigma_{k}+V\left(-T_{k-1}^{i j} \nabla_{i} \nabla_{j} F-F\left(\sigma_{1} \sigma_{k}-(k+1) \sigma_{k+1}\right)+(n-k) F \sigma_{k-1}+F \sigma_{1} \sigma_{k}\right)\right\} d \mu \\
& =\int_{\Sigma}\left(u F \sigma_{k}-V\left(T_{k-1}^{i j} \nabla_{i} \nabla_{j} F-F(n-k) \sigma_{k-1}\right)+(k+1) V \sigma_{k+1} F\right) d \mu \\
& =\int_{\Sigma}\left(u F \sigma_{k}-\left(\nabla_{j}\left(T_{k-1}^{i j} \nabla_{i} V\right)-(n-k) \sigma_{k-1} V-(k+1) V \sigma_{k+1}\right) F\right) d \mu \\
& =\int_{\Sigma}(k+1)\left(u \sigma_{k}+V \sigma_{k+1}\right) F d \mu
\end{aligned}
$$

here we used the fact $T_{k-1}$ is divergence-free and in the fifth equality we used (7.2). Thus

$$
\frac{\partial}{\partial t} \int_{\Sigma} V p_{k} d \mu=\int_{\Sigma}\left((k+1) u p_{k}+(n-k-1) V p_{k+1}\right) F d \mu .
$$

The proof of (5) follows from a similar computation.

In order to show that $E$ is non-negative, we use the following flow

$$
\frac{\partial X}{\partial t}=-V \nu
$$

which was first used in [11].

Theorem 8.2. Let $1 \leq k<n-1$. Any horospherical convex hypersurface in the hyperbolic space $\mathbb{H}^{n}$ satisfies

$$
\int_{\Sigma} V p_{k+1} \geq \int_{\Sigma}\left(V p_{k-1}+\frac{p_{k+1}}{V}\right) d \mu .
$$


By Lemma 8.1, one immediately obtains the evolution equation of $E$ along flow (8.4),

$$
\begin{aligned}
\frac{d E}{d t}= & \int_{\Sigma}\left\{\left((k+2) u p_{k+1}+(n-k-2) V p_{k+2}\right)(-V)-\left(k u p_{k-1}+(n-k) V p_{k}\right)(-V)\right\} d \mu \\
& +\int_{\Sigma}\left(k \frac{u p_{k+1}}{V}+(n-k-2) p_{k+2}\right) d \mu \\
= & -(n-k-2) \int_{\Sigma}\left(V^{2} p_{k+2}-V^{2} p_{k}-p_{k+2}\right) d \mu-2 \int_{\Sigma}\left(u V p_{k+1}-V^{2} p_{k}\right) d \mu \\
& -k \int_{\Sigma}\left(u V p_{k+1}-u V p_{k-1}-\frac{u p_{k+1}}{V}\right) d \mu .
\end{aligned}
$$

It follows, together with (7.6) and Proposition 7.4 that the monotonicity of the functional $E$

$$
\frac{d E}{d t} \leq 0
$$

along the flow (8.4). That is, we have showed that

Proposition 8.3. The functional $E$ defined in (8.1) is non-increasing under flow 8.4).

For the proof Theorem 8.2 , we need the following two more lemmas.

Lemma 8.4. Flow 8.4 preserves the horospherical convexity.

Proof. A direct computation gives (or see [43] for instance)

$$
\partial_{t} h_{j}^{i}=\nabla^{i} \nabla_{j} V+V\left(\left(h^{2}\right)_{j}^{i}-\delta_{j}^{i}\right) .
$$

Set

Noting that the fact

$$
\widetilde{h}_{j}^{i}:=h_{j}^{i}-\delta_{j}^{i},
$$

$$
\nabla^{i} \nabla_{j} V=V \delta_{j}^{i}-u h_{j}^{i}
$$

which follows from (7.1), one has

$$
\partial_{t} \widetilde{h}_{j}^{i}=(V-u) \delta_{j}^{i}+V\left(\left(\widetilde{h}^{2}\right)_{j}^{i}+2(\widetilde{h})_{j}^{i}\right)-u \widetilde{h}_{j}^{i} .
$$

Let $a$ be a unit vector such that

$$
\widetilde{h}_{j}^{i} a^{j}=0 .
$$

Then we have

$$
\left((V-u) \delta_{j}^{i}+V\left(\left(\widetilde{h}^{2}\right)_{j}^{i}+2(\widetilde{h})_{j}^{i}\right)-u \widetilde{h}_{j}^{i}\right) a_{i} a^{j}=V-u>0 .
$$

The Lemma follows easily.

Lemma 8.5. The quermassintegrals are monotone under the set inclusion, i.e.

$$
W_{k}\left(K_{1}\right) \leq W_{k}\left(K_{2}\right), \quad \text { if } K_{1} \subset K_{2} \text {. }
$$

As a consequence,

$$
\int_{\partial K_{1}} \sigma_{k} d \mu \leq \int_{\partial K_{2}} \sigma_{k} d \mu, \quad \text { if } K_{1} \subset K_{2} .
$$

Proof. (8.7) follows easily from the definition of the quermassintegral, see (6.12). (8.8) follows from (8.7) and (6.13) 
Proof Theorem 8.2, For any horospherical convex hypersurface $\Sigma$ we consider the flow with $F=-V$. Let $T^{*} \in(0, \infty)$ be the maximal time of existence of the flow. It is clear that $T^{*}$ is finite. Let $\Sigma_{t}$ be the evolved hypersurface for $t \in\left[0, T^{*}\right)$ and define $r(t)$ and $R(t)$ the inner radius and outer radius of $\Sigma_{t}$ respectively. It is clear that $r(t) \rightarrow 0$ when $t \rightarrow T^{*}$. By Lemma 8.4 we know that every $\Sigma_{t}$ is horospherical convex. As a feature of horospherical convex hypersurface, $R(t)$ is controlled by $3 \sqrt{r(t)}$ from above when $r(t)$ is sufficiently small, and hence we have that $R(t)$ also converges to 0 , as $t \rightarrow T^{*}$. See [6]. From the monotonicity (8.8), we have

$$
\int_{\Sigma_{t}} \sigma_{k} d \mu \leq \int_{\partial B_{R(t)}} \sigma_{k} d \mu
$$

where $B_{R(t)}$ and $\partial B_{R(t)}$ are the geodesic ball, the geodesic sphere of radius $R(t)$ respectively, for $\Sigma_{t} \subset B_{R(t)}$. It is easy to check that

$$
\int_{\Sigma_{t}} \sigma_{k} d \mu \leq \int_{\partial B_{R(t)}} \sigma_{k} d \mu \rightarrow 0, \quad \text { as } R(t) \rightarrow 0 .
$$

It follows readily that

$$
\int_{\Sigma_{t}} V^{l} \sigma_{k} d \mu \rightarrow 0
$$

which implies that $E\left(\Sigma_{t}\right) \rightarrow 0$. By Proposition 8.3 we have $E(\Sigma) \geq 0$.

We remark that here we have used the horospherical convexity. We believe that in the argument the convexity should be enough, if we use a more precise information about the conformal flow (8.4).

\section{Weighted Alexandrov-Fenchel inequalities}

Now we begin to prove Theorem 6.2 ,

Proof of Theorem 6.2. Due to Theorem 8.2, we are able to use the induction argument to prove this theorem. When $k=0$, (6.9) is just (6.8), which was proved in [26]. Assume (6.9) holds for $k-1$, namely the following holds,

$$
\int_{\Sigma} V p_{2 k-1} d \mu \geq \omega_{n-1}\left\{\left(\frac{|\Sigma|}{\omega_{n-1}}\right)^{\frac{n}{k(n-1)}}+\left(\frac{|\Sigma|}{\omega_{n-1}}\right)^{\frac{n-2 k}{k(n-1)}}\right\}^{k} .
$$

We need to show that (6.9) holds for $k$. For the simplicity of notation we denote

$$
\|\Sigma\|=\frac{|\Sigma|}{\omega_{n-1}}
$$

First recall (6.5) that

$$
\int_{\Sigma} p_{2 k+1} d \mu \geq \omega_{n-1}\left(\|\Sigma\|^{\frac{2}{2 k+1}}+\|\Sigma\|^{\frac{2(n-2 k-2)}{(2 k+1)(n-1)}}\right)^{\frac{2 k+1}{2}} .
$$


It follows, together with the Hölder inequality, that

$$
\begin{aligned}
\left(\int_{\Sigma} V p_{2 k+1} d \mu\right)\left(\int_{\Sigma} \frac{p_{2 k+1}}{V} d \mu\right) & \geq\left(\int_{\Sigma} p_{2 k+1} d \mu\right)^{2} \\
& \geq \omega_{n-1}^{2}\left(\|\Sigma\|^{\frac{2}{2 k+1}}+\|\Sigma\|^{\frac{2(n-2 k-2)}{(2 k+1)(n-1)}}\right)^{2 k+1} \\
& =\omega_{n-1}^{2}\|\Sigma\|^{2}\left(1+\|\Sigma\|^{-\frac{2}{n-1}}\right)^{2 k+1} .
\end{aligned}
$$

Set

$$
\alpha:=\omega_{n-1}^{2}\|\Sigma\|^{2}\left(1+\|\Sigma\|^{-\frac{2}{n-1}}\right)^{2 k+1}
$$

From above we have

$$
\int_{\Sigma} V p_{2 k+1} d \mu-\int_{\Sigma} \frac{p_{2 k+1}}{V} d \mu \leq \int_{\Sigma} V p_{2 k+1} d \mu-\frac{\alpha}{\int_{\Sigma} V p_{2 k+1} d \mu} .
$$

From our crucial Minkowski integral formula (8.5) and the induction assumption (9.1), we have

$$
\begin{aligned}
\int_{\Sigma} V p_{2 k+1} d \mu-\int_{\Sigma} \frac{p_{2 k+1}}{V} d \mu & \geq \int_{\Sigma} V p_{2 k-1} d \mu \\
& \geq \omega_{n-1}\|\Sigma\|^{\frac{n}{n-1}}\left(1+\|\Sigma\|^{-\frac{2}{n-1}}\right)^{k} .
\end{aligned}
$$

We introduce an auxiliary function $f(t):=t-\frac{\alpha}{t}$. Then (9.2) and (9.3) imply

$$
f\left(\int_{\Sigma} V p_{2 k+1} d \mu\right) \geq \omega_{n-1}\|\Sigma\|^{\frac{n}{n-1}}\left(1+\|\Sigma\|^{-\frac{2}{n-1}}\right)^{k} .
$$

On the other hand, one can easily check that

$$
\begin{aligned}
& f\left(\omega_{n-1}\left(\|\Sigma\|^{\frac{n}{(k+1)(n-1)}}+\|\Sigma\|^{\frac{n-2 k-2}{(k+1)(n-1)}}\right)^{k+1}\right) \\
= & \omega_{n-1}\|\Sigma\|^{\frac{n}{n-1}}\left(1+\|\Sigma\|^{-\frac{2}{n-1}}\right)^{k+1}-\omega_{n-1}\|\Sigma\|^{\frac{n-2}{n-1}}\left(1+\|\Sigma\|^{-\frac{2}{n-1}}\right)^{k} \\
= & \omega_{n-1}\|\Sigma\|^{\frac{n}{n-1}}\left(1+\|\Sigma\|^{-\frac{2}{n-1}}\right)^{k} \\
\leq & f\left(\int_{\Sigma} V p_{2 k+1} d \mu\right) .
\end{aligned}
$$

The last inequality follows from (9.4). Since $f$ is increasing on the interval $[0,+\infty)$, we have

$$
\int_{\Sigma} V p_{2 k+1} d \mu \geq \omega_{n-1}\left(\|\Sigma\|^{\frac{n}{(k+1)(n-1)}}+\|\Sigma\|^{\frac{n-2 k-2}{(k+1)(n-1)}}\right)^{k+1}
$$

the desired result (6.9). When equality holds, it follows from the equality in Hölder inequality that $V$ is constant on $\Sigma$, which yields $\Sigma$ is a centered geodesic sphere. Hence we complete the proof. $k$.

We end this section with a conjecture of "weighted" Alexandrov-Fenchel inequalities for even 
Conjecture 9.1. Let $k \leq n-1$ be even. Any horospherical convex hypersurface $\Sigma$ in the hyperbolic space $\mathbb{H}^{n}$ satisfies

$$
\int_{\Sigma} V \sigma_{k} d \mu \geq C_{n-1}^{k} \omega_{n-1}\left(\left(\frac{|\Sigma|}{\omega_{n-1}}\right)^{\frac{2 n}{(k+1)(n-1)}}+\left(\frac{|\Sigma|}{\omega_{n-1}}\right)^{\frac{2(n-k-1)}{(k+1)(n-1)}}\right)^{\frac{k+1}{2}} .
$$

Equality holds if and only if $\Sigma$ is a centered geodesic sphere in $\mathbb{H}^{n}$.

We have proved inequality (9.5) for odd $k$. For even $k$ our argument presented in this paper still works if the induction argument could start, i.e., if the following inequality

$$
\int_{\Sigma} V d \mu \geq \omega_{n-1}\left(\left(\frac{|\Sigma|}{\omega_{n-1}}\right)^{\frac{2 n}{n-1}}+\left(\frac{|\Sigma|}{\omega_{n-1}}\right)^{2}\right)^{\frac{1}{2}}
$$

holds. We believe that (9.6) is true. However, we could not prove it yet. Instead, we have a weaker version of (9.5) for even $k$, which is also optimal.

Theorem 9.2. Any horospherical convex hypersurface $\Sigma$ in the hyperbolic space $\mathbb{H}^{n}$ satisfies

$$
\int_{\Sigma} V \sigma_{2 k} d \mu \geq C_{n-1}^{2 k} \omega_{n-1}\left(\left(\frac{\int_{\Sigma} u d \mu}{\omega_{n-1}}\right)^{\frac{2}{2 k+1}}+\left(\frac{\int_{\Sigma} u d \mu}{\omega_{n-1}}\right)^{\frac{2(n-2 k-1)}{(2 k+1) n}}\right)^{\frac{2 k+1}{2}}
$$

Here $u=\langle\bar{\nabla} V, \nu\rangle>0$ is the support function, where $\nu$ is the outer normal vector. Equality holds if and only if $\Sigma$ is a centered geodesic sphere in $\mathbb{H}^{n}$.

Proof. As in the proof of Theorem 6.2, we adopt the induction argument. First when $k=0$, it follows from (7.3) and Hölder's inequality that

$$
\begin{aligned}
\left(\int_{\Sigma} V d \mu\right)^{2} & \geq\left(\int_{\Sigma} V d \mu\right)\left(\int_{\Sigma} \frac{1}{V} d \mu+\int_{\Sigma} \frac{u^{2}}{V} d \mu\right) \\
& \geq\left(\int_{\Sigma} 1 d \mu\right)^{2}+\left(\int_{\Sigma} u d \mu\right)^{2} .
\end{aligned}
$$

Using the fact

$$
\left(\frac{|\Sigma|}{\omega_{n-1}}\right)^{\frac{n}{n-1}} \geq \frac{\int_{\Sigma} u d \mu}{\omega_{n-1}}
$$

which has been proved in [26] (see Proposition 3.3), we obtain the desired result for $k=0$ that

$$
\left(\int_{\Sigma} V d \mu\right)^{2} \geq \omega_{n-1}^{2}\left(\left(\frac{\int_{\Sigma} u d \mu}{\omega_{n-1}}\right)^{2}+\left(\frac{\int_{\Sigma} u d \mu}{\omega_{n-1}}\right)^{\frac{2(n-1)}{n}}\right)
$$

Now we can start the induction argument. Noting that by (6.5) and (9.8), we have

$$
\begin{aligned}
\int_{\Sigma} \sigma_{k} d \mu & \geq C_{n-1}^{k} \omega_{n-1}\left\{\left(\frac{\int_{\Sigma} u d \mu}{\omega_{n-1}}\right)^{\frac{2(n-1)}{k n}}+\left(\frac{\int_{\Sigma} u d \mu}{\omega_{n-1}}\right)^{\frac{2(n-k-1)}{k n}}\right\}^{\frac{k}{2}} \\
& =C_{n-1}^{k} \omega_{n-1}\left(\frac{\int_{\Sigma} u d \mu}{\omega_{n-1}}\right)^{\frac{n-1}{n}}\left\{1+\left(\frac{\int_{\Sigma} u d \mu}{\omega_{n-1}}\right)^{-\frac{2}{n}}\right\}^{\frac{k}{2}} .
\end{aligned}
$$


The rest of the proof is essentially the same as in the one of Theorem 6.2 and we skip it.

\section{REFERENCES}

[1] L. J. Alías, J. H. S. de Lira and J. M. Malacarne, Constant higher-order mean curvature hypersurfaces in Riemannian spaces, J. Inst. Math. Jussieu, 5 (2006), no. 4, 527-562.

[2] L. Andersson, M. Cai, and G. J. Galloway, Rigidity and positivity of mass for asymptotically hyperbolic manifolds, Ann. Henri Poincaré 9 (2008), no. 1, 1-33.

[3] R. Arnowitt, S. Deser and C.W. Misner, Coordinate invariance and energy expressions in general relativity, Phys. Rev. (2) 122, 997-1006, (1961).

[4] R. Bartnik, The mass of an asymptotically flat manifold, Comm.Pure Appl.Math. 34, 661-693, (1986).

[5] W. Becker, Sharp Sobolev inequalities on the sphere and the Moser-Trudinger inequality, Ann. of Math. 138, 213-242(1993).

[6] A. A. Borisenko and V. Miquel, Total curvatures of convex hypersurfaces in hyperbolic space, Illinois J. Math. 43 (1999), 61-78.

[7] V. Bonini and J. Qing, A positive mass theorem on asymptotically hyperbolic manifolds with corners along a hypersurface. Ann. Henri Poincaré 9 (2008), no. 2, 347-372.

[8] H. L. Bray, Proof of the Riemannian Penrose inequality using the positive mass theorem, J. Differential Geom. 59 (2001), no. 2, 177-267.

[9] H. L. Bray, On the positive mass, Penrose, an ZAS inequalities in general dimension, Surveys in Geometric Analysis and Relativity, Adv. Lect. Math. (ALM), 20, Int. Press, Somerville, MA, (2011).

[10] H. L. Bray and D. A. Lee, On the Riemannian Penrose inequality in dimensions less than eight, Duke Math. J. 148 (2009), no. 1, 81-106.

[11] S. Brendle, Hypersurfaces of constant mean curvature in deSitter-Schwarzschild space, arXiv:1105.4273.

[12] S. Brendle, P.-K. Hung and M.-T. Wang, A Minkowski-type inequality for hypersurfaces in the AntideSitter-Schwarzschild manifold, arXiv: 1209.0669.

[13] Y. D. Burago and V. A. Zalgaller, Geometric Inequalities, Springer, Berlin, (1988).

[14] T. Caúla, L. L. de Lima and N. L. Santos, Deformation and rigidity results for the $2 k$-Ricci tensor and the $2 k$-Gauss-Bonnet curvature, arXiv:math/12081801v1.

[15] S.-Y. A. Chang and Y.Wang, On Aleksandrov-Fenchel inequalities for k-convex domains, Milan J. Math., 79 (2011), no. 1, 13-38.

[16] X. Cheng and D. Zhou, Rigidity for nearly umbilical hypersurfaces in space forms, arXiv:1208.1786.

[17] S. S. Chern, A simple intrinsic proof of the Gauss-Bonnet formula for closed Riemannian manifolds, Ann. of Math. (2) 45 (1944). 747-752.

[18] S. S. Chern, On the curvatura integra in a Riemannian manifold, Ann. of Math. (2) 46 (1945) 674-684.

[19] P. Chruściel and M. Herzlich, The mass of asymptotically hyperbolic Riemannian manifolds, Pacific J. Math. 212 (2003), no. 2, 231-264.

[20] P. Chruściel and G. Nagy, The mass of spacelike hypersurface in asymptotically anti-de Sitter space-times, Adv. Theor. Math. Phys. 5 (2002), 697-754 gr-qc/0110014

[21] J. Crisóstomo, R. Troncoso and J. Zanelli, Black hole scan, PHys.Rev.D(3) 62 (2000), no.8, 084013.

[22] M. Dahl, R. Gicquaud and A. Sakovich, Penrose type inequalities for asymptotically hyperbolic graphs, Annales Henri Poincaré (2012), DOI 10.1007/s00023-012-0218-4.

[23] X. Dai, A positive mass theorem for spaces with asymptotic SUSY compactification, Commun. Math. Phys. 244 (2004) 335-345.

[24] L. L. de Lima and F. Girão, The ADM mass of asymptotically flat hypersurfaces, arXiv:1108.5474.

[25] L. L. de Lima and F. Girão, Positive mass and Penrose type inequalities for asymptotically hyperbolic hypersurfaces, arXiv:1201.4991.

[26] L. L. de Lima and F. Girão, An Alexandrov-Fenchel-type inequality in hyperbolic space with an application to a Penrose inequality, arXiv:12090669.

[27] S. Deser and B. Tekin, Gravitational energy in quadratic-curvature gravities Physical review letters, 89 (2002) 101101.

[28] S. Deser and B. Tekin, Energy in generic higher curvature gravity theories, Physical Review D, 75 (2003) 084032 . 
[29] E. Gallego and G. Solanes, Integral geometry and geometric inequalities in hyperbolic space, Differential Geom. Appl. 22 (2005), 315-325.

[30] Y. Ge, G. Wang and J. Wu, A new mass for asymptotically flat manifolds, arXiv:1211.3645.

[31] Y. Ge, G. Wang and J. Wu, The Gauss-Bonnet-Chern mass of conformally flat manifolds, arXiv:1212.3213. to appear in IMRN.

[32] Y. Ge, G. Wang and J. Wu, Hyperbolic Alexandrov-Fenchel quermassintegral inequalities I, arXiv:1303.1714.

[33] Y. Ge, G. Wang and J. Wu, Hyperbolic Alexandrov-Fenchel quermassintegral inequalities II, arXiv:1304.1417.

[34] C. Gerhardt, Inverse curvature flows in hyperbolic space, J. Differential Geom. 89 (2011), no. 3, $487-527$.

[35] P. Guan, Curvature measures, isoperimetric type inequalities and fully nonlinear PDES, Lecture notes.

[36] P. Guan and J. Li, The quermassintegral inequalities for k-convex starshaped domains, Adv. Math. 221 (2009), 1725-1732.

[37] P. Guan and J. Li, A mean curvature flow in space forms, preprint.

[38] P. Guan and J. Li, in preparation.

[39] M. Herzlich, A Penrose-like inequality for the mass of Riemannian asymptotically flat manifolds, Comm. Math. Phys., 188 (1997), 121-133.

[40] M. Herzlich, Mass formulae for asymptotically hyperbolic manifolds, AdS/CFT correspondence: Einstein metrics and their conformal boundaries, 103-121, Eur. Math. Soc., Zürich, (2005).

[41] L.-H. Huang and D. Wu, The equality case of the Penrose inequality for asymptotically flat graphs, arXiv:1205.2061

[42] L.-H. Huang and D. Wu, Hypersurfaces with nonnegative scalar curvature, arXiv.1102.5749.

[43] G. Huisken, Flow by mean curvature of convex surfaces into spheres, J. Differential Geom. 20 (1984) $237-266$.

[44] G. Huisken, in preparation. See also 36.

[45] G. Huisken and T. Ilmanen, The inverse mean curvature flow and the Riemannian Penrose inequality, J. Diff. Geom. 59 (2001), 353-437.

[46] M.L. Labbi, On (2k)-minimal submanifolds, Result.Math. 52, (2008), 323-338.

[47] M.-K. G. Lam, The graph cases of the Riemannian positive mass and Penrose inequality in all dimensions, arXiv.org/1010.4256.

[48] C. Lanczos, A remarkable property of the Riemann-Christoffel tensor in four dimensions, Ann. of Math. (2) 39 (1938), no. 4, 842-850.

[49] D. Lee and A. Neves, A static uniqueness theorem for higher genus Kottler metrics, slides of a talk delivered at Tsinghua Sanya International Mathematical Forum.

[50] Y. Li and L. Nguyen, A generalized mass involving higher order symmetric function of the curvature tensor, arXiv:1211.3676.

[51] H. Li, Y. Wei and C. Xiong, A geometric ineqality on hypersurface in hyperbolic space, arXiv:1211.4109.

[52] J. Lohkamp, The Higher Dimensional Positive Mass Theorem I, arXiv:0608795.

[53] D. Lovelock, The Einstein tensor and its generalizations J. Math. Phys. 12 (1971) 498-501.

[54] M. Mars, Topical review: Present status of the Penrose inequality, Classical and Quantum Gravity, 26 (2009), no.19, 193001.

[55] P. Miao, Positive mass theorem on manifolds admitting corners along a hypersurface, Adv. Theor. Math. Phys. 6 (2002), no. 6, 1163-1182.

[56] B. Michel, Geometric invariance of mass-like asymptotic invariants, J. Math. Phys. 52 (2011), no. 5.

[57] A. Neves, Insufficient convergence of inverse mean curvature flow on asymptotically hyperbolic manifolds, J. Diff. Goem. 84, 191-229 (2010).

[58] T. Parker and C. Taubes, On Witten's proof of the positive energy theorem, Comm. Math. Phys., 84 (1982), 223-238.

[59] E. M. Patterson, A class of critical Riemannian metrics, J. London Math. Soc. (2) 23 (1981), no. 2, 349-358.

[60] R. C. Reilly, On the Hessian of a function and the curvatures of its graph, Michigan Math. J. 20, (1973), 373-383.

[61] R. C. Reilly, Variational properties of functions of the mean curvatures for hypersurfaces in space forms, J.Differential Geometry, 8 (1973) 465-477. 
[62] I. Rivin and Jean-Marc Schlenker, On the Schlafli differential formula, arXiv:math/0001176

[63] L. Santaló, Integral geometry and geometric probability, Cambridge Mathematical Library. Cambridge University Press, Cambridge, (2004).

[64] E. Schmidt, Die isoperimetrischen Ungleichungen auf der gewöhnlichen Kugel und für Rotationskörper im n-dimensionalen sphärischen Raum. (German) Math. Z. 46, (1940), 743-794.

[65] R. Schneider, Convex bodies: The Brunn-Minkowski theory, Cambridge University, (1993), MR1216521.

[66] R. Schoen, talk at the Simons Center for Geometry and Physics, November, (2009).

[67] R. Schoen and S. T. Yau, On the proof of the positive mass conjecture in general relativity, Comm. Math. Phys. 65 (1979), 45-76.

[68] R. Schoen and S. T. Yau, Conformally flat manifolds, Kleinian groups and scalar curvature, Invent. math., 92.

[69] F. Schwartz, A volumetric Penrose inequality for conformally flat manifolds, Ann. Henri Poincaré 12 (2011), no. 1, 67-76.

[70] Y. Shi, T.-F. Tam, Positive mass theorem and the boundary behaviors of compact manifolds with nonnegative scalar curvature, J. Diff. Geom. 62 (2002) 79-125.

[71] G. Solanes, Integral geometry and the Gauss-Bonnet theorem in constant curvature spaces, Trans. Amer. Math. Soc. 358 (2006), no. 3, 1105-1115.

[72] X. Wang, Mass for asymptotically hyperbolic manifolds, J.Diff.Geom. 57 (2001), 273-299.

[73] G. Wang and C. Xia, Isoperimetric type problems and Alexandrov-Fenchel type inequalities in the hyperbolic space, arXiv:1304.1674.

[74] A. Willa, Dimensionsabhängige Relationen für den Krümmungstensor und neue Klassen von Einsteinund Spuereinsteinräumen, Diss ETH Nr. 14026,

[75] E. Witten, A new proof of the positive energy theorem, Comm. Math. Phys., 80 (1981) 381-402.

[76] W. Zhang, Lectures on Chern-Weil theory and Witten deformations, Nankai Tracts in Mathematics, 4 World Scientific Publishing Co., Inc., River Edge, NJ, (2001).

[77] X. Zhang, A definition of total energy-momenta and the positive mass theorem on asymptotically hyperbolic 3-manifolds. I. Comm. Math. Phys. 249 (2004), no. 3, 529-548.

Laboratoire D'Analyse et de Mathématiques Appliquées, CNRS UMR 8050, Département de Mathématiques, Université Paris Est-Créteil Val de Marne,, 61 avenue du Général de Gaulle, 94010 Créteil Cedex, France

E-mail address: ge@u-pec.fr

Albert-Ludwigs-Universität Freiburg, Mathematisches Institut Eckerstr. 1 D-79104 Freiburg

E-mail address: guofang.wang@math.uni-freiburg.de

School of Mathematical Sciences, University of Science and Technology of China Hefei 230026, P. R. China and Albert-Ludwigs-Universität Freiburg, Mathematisches Institut Eckerstr. 1 D79104 FrEIBURG

E-mail address: jie.wu@math.uni-freiburg.de 